\title{
An annotated checklist of Dolichopodidae (Diptera) of Yakutia (Siberia) with some new records
}

\author{
Аннотированный список видов семейства Dolichopodidae \\ (Diptera) Якутии и новые указания
}

\author{
I.Ya. Grichanov ${ }^{*}$, A.K. Bagachanova ${ }^{2}$ \\ И.Я. Гричанов ${ }^{1}$, А.К. Багачанова ${ }^{2}$
}

\footnotetext{
1 All-Russian Institute of Plant Protection, Podbelskogo 3, St. Petersburg-Pushkin, 189620, Russia. Всероссийский институт защиты растений, шоссе Подбельского 3, Санкт-Петербург-Пушкин 196608, Россия.

* Corresponding author, E-mail: grichanov@mail.ru

${ }^{2}$ Institute for Biological Problems of Cryolithozone SD RAS, Lenin Avenue, 41, Yakutsk, 677007, Russia. E-mail: a.k.bag@ibpk.ysn.ru Институт биологических проблем криолитозоны CO РАН, просп. Ленина, 41, Якутск, 677007, Россия.
}

KEY WORDS: Dolichopodidae, Russia, Yakutia, checklist, new records.

КЛЮЧЕВЫЕ СЛОВА: Dolichopodidae, Россия, Якутия, список видов, новые указания.

ABSTRACT. A new material of Dolichopodidae was recently collected and/or identified, and a revised check-list of Yakutian species was compiled. The present research gives new records, including 28 species found for the first time in Yakutia, 31 species recorded from new natural zones and four probably undescribed species. In total, 20 genera and 150 (including four unnamed) species are listed that apparently makes up 60$70 \%$ of actual Dolichopodidae fauna in Yakutia. This paper provides also distribution pattern for each species and characteristics of Yakutian habitats for 54 species.

How to cite this article: Grichanov I.Ya., Bagachanova A.K. 2018. An annotated checklist of Dolichopodidae (Diptera) of Yakutia (Siberia) with some new records // Russian Entomol. J. Vol.27. No.1. P.73-92. doi: $10.15298 /$ rusentj.27.1.12

РЕЗЮМЕ. Новый материал по семейству Dolichopodidae Якутии собран и/или определен; составлен новый список видов Республики. Новые указания включают 28 видов, найденных впервые в Якутии, 31 вид, обнаруженный в новых природных зонах, и четыре, скорее всего, неописанных вида. Всего в Республике отмечено 20 родов и 150 видов, что, по-видимому, составляет $60-70 \%$ якутской фауны Dolichopodidae. В статье приведены также общее распространение для каждого вида и краткие характеристики мест обитания для 54 видов, отловленных в Якутии.

\section{Introduction}

Yakutia (presently, the official name of this land is "Sakha (Yakutia) Republic") is a vast (more than $3000000 \mathrm{~km}^{2}$ ) and scarcely populated (about 1 million) region situated in NE Asia and characterized by a severe continental climate with the absolute annual amplitude of temperature reaching $101.8^{\circ} \mathrm{C}$ at Verkhoyansk [Suslov, 1999]. Most of its territory, except for high mountains of its NE part, is covered by open taiga of lowgrowing larch (Larix spp.) with admixture of pine ( $P i$ nus silvestris) and birch (Betula pendula s. 1.). The forests exist there in spite of extremely low precipitation (mostly in late summer, annual precipitation ranging from 90 to $300 \mathrm{~mm}$ ), which is compensated by a low evaporation. The summer is quite short (80-110 days without frost) but sunny and hot (at Yakutsk, the average temperature of July is $19^{\circ} \mathrm{C}$, the maximum temperature registered is $38^{\circ} \mathrm{C}$ ); so, many animal species extend more northerly in Yakutia than in West Siberia [Suslov, 1999]. Rather high latitude provides a very long daytime in June and July and hence a rapid passing of all phenological phases in the beginning of the summer; the best time to collect almost all insects there is June [see Suslov, 1999 and Kuznetsova, 2005 for further reading].

The earliest collection of Yakutian Dolichopodidae was made by the Russian researcher of Siberia Aleksander Czekanowski in 1874-1875 in the Far North of Yakutia during his expeditions along the Olenyok River mainly. The material was treated in $20^{\text {th }}$ century, being still kept in the Zoological institute (ZIN) of the Russian Academy of Sciences (St.-Petersburg).

The first information about Yakutian long-legged flies was published by the Finnish researcher R. Frey [Frey, 1915] who treated Diptera material collected during the 1900-1902 Russian polar expedition and found Hydrophorus alpinus Wahlberg, 1844. The results of treatment of rich dolichopodid materials collected by the Yakutian expedition of the Academy of Sciences of the USSR (1925-1927) were published in a series of works of the famous Soviet dipterologist 
A.A. Stackelberg [1928, 1929, 1930a, b, 1933, 1951]. Since 1950s, the ZIN dolichopodid collection replenished by various expeditions to Yakutia. The very interesting findings were made by well known dipterists K.B. Gorodkov and V.G. Kovalyov in the northern, northeastern and central parts of the Republic, by V.V. Zlobin in the Center, West and Southwest. These materials were used in a number of taxonomical revisions and faunistic reviews of dolichopodid fauna of the USSR and Russia published by O.P. Negrobov and his disciples since 1971 (see References). The Catalogue for the family Dolichopodidae (Diptera) in the USSR that presented an annotated list of species with region by region records contained 45 species known for Yakutia [Grichanov, Negrobov, 1979]. Later, several more species records were published in taxonomical and faunistic works based mainly on ZIN collections [Negrobov, 1986a, b, 2000; Negrobov, Tsurikov, 1986; Negrobov, Chalaya, 1991; Negrobov, Maslova, 1995; etc.].
Recently, rich Diptera material was accumulated at the Institute of Biological Problems of the Cryolythozone of the Siberian Branch of the Russian Academy of Sciences, Yakutsk (IBPC). The flies collected before 2002 were studied by I.Ya. Grichanov, who found 11 species new for the fauna of Yakutia and compiled a check-list for the Republic for the first time, including 76 species [Grichanov, 2002]. New data on the distribution of 51 species of Yakutian Dolichopodidae including 17 species new for the Republic were published in 2006 [Grichanov, Bagachanova, 2006]. Last years, Grichanov, Volfov [2009]; Maslova et al. [2011]; Negrobov, Grichanov [2013], Grichanov, Negrobov [2014]; Negrobov et al. [2017] have described several new for science species from that territory. At the same time, A.K. Bagachanova and her collaborators (IBPC) have collected a lot of new material from various parts of Yakutia, and I.Ya. Grichanov has identified recently sorted flies from the old ZIN and IBPC collections.

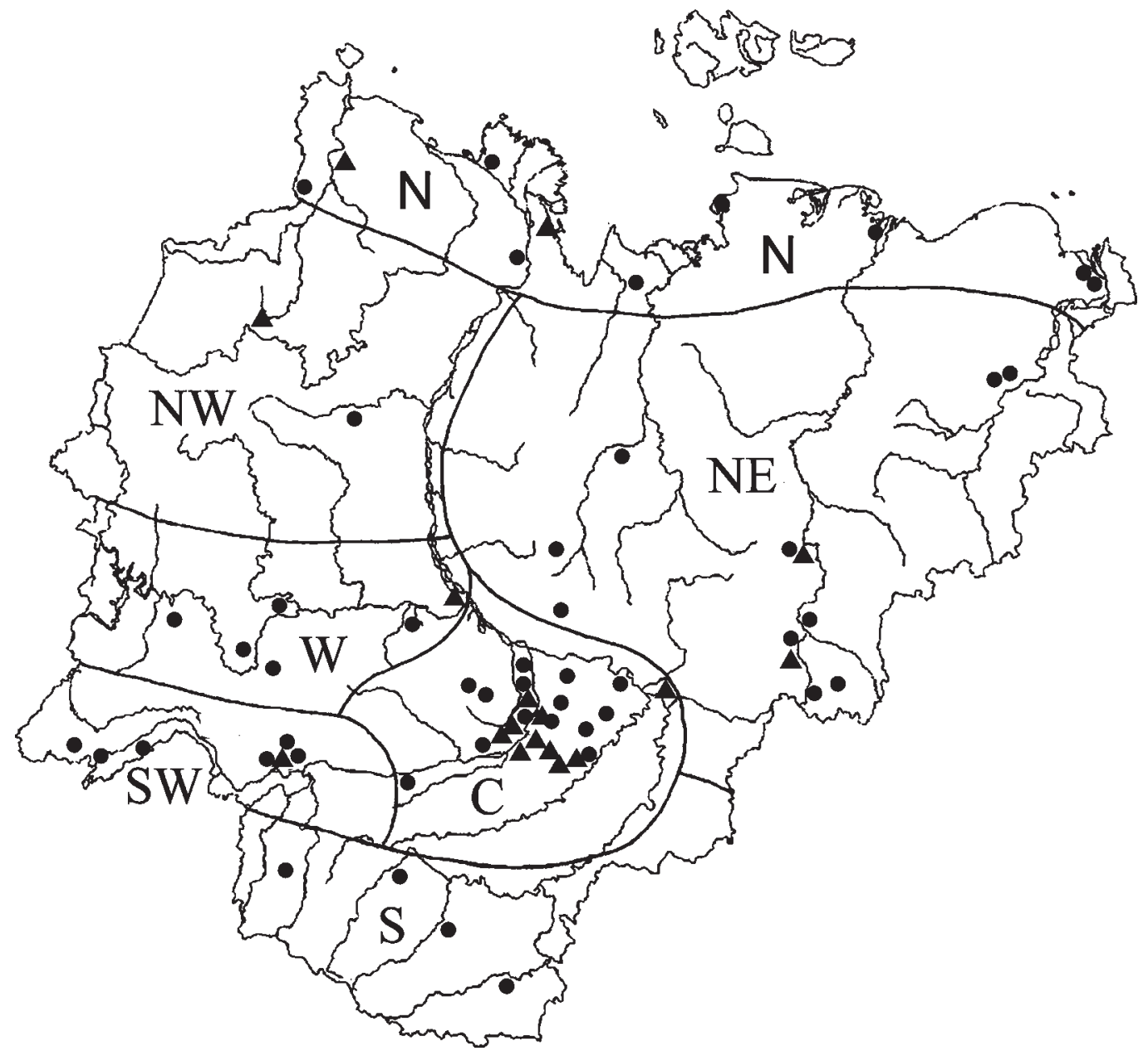

Fig. 1. Points of collecting dolichopodids in Yakutia: - listed by authors [Grichanov, Bagachanova, 2006, and this paper]; $\mathbf{\Delta}$ according with other references. The following designations were adopted in the text and Figure 1: N — North Yakutia; NW - North-West Yakutia; NE - North-East Yakutia; W - West Yakutia; C - Central Yakutia; SW - South-West Yakutia; S - South Yakutia [after Vinokurov et al., 2010].

Рис. 1. Места находок видов Dolichopodidae в Якутии: - указанные авторами [Grichanov, Bagachanova, 2006, и настоящая статья]; $\mathbf{\Delta}$ - приведённые по другим источникам. Обозначения, использованные на рис. 1 и в тексте: N - Cеверная Якутия; NW - Север-западная Якутия; NE - Северо-восточная Якутия; W - Западная Якутия; C - Центральная Якутия; SW - Юго-западная Якутия; S - Южная Якутия [по Винокурову и др., 2010]. 
It is worth noting that the papers based on old collections contained a lot of missing data, inaccuracies and wrong spacing of collection points. Distribution of species was sometimes provided in general, e.g. "Yakutia", "Indigirka". In some cases, localities were erroneously associated with the recognized natural regions of the Republic, e.g. with the East Yakutia instead of the Central Yakutia. For this paper, the authors have checked original (label) localities, if they were referenced, and established the exact locations of collection points according with primary sources (Fig. 1). Such work is necessary for bonding a fly distribution with the landscape and vegetation zones of Yakutia [after Vinokurov et al., 2010]. In future, it will allow characterizing dolichopodid fly species by area types [Gorodkov, 1984].

\section{Annotated List of Species}

For each species we provide references (if available), with natural zones of Yakutia (Fig. 1) for the published material and with the names of taxa used by the respective authors if they differ from those used by us; unpublished material studied; short characteristics of species habitats (if available); general distribution of species [after Grichanov, 2017] and preliminary characteristics of a species area type [after Gorodkov, 1984]. An asterisk (*) indicates new species for the Republic. Remarks are provided where deemed necessary.

\section{Argyra magnicornis (Zetterstedt, 1838)*}

MATERIAL EXAMINED. C Yakutia: $10^{7}$, Amginsky District, Mikhailovka, 25.07.1987, Zlobin (ZIN).

DISTRIBUTION. Type locality: Norway: "Nordlandiae, Tromsoe, Lappon. Norveg." Palaearctic: Austria, Denmark, Finland, France, Germany, Norway, Russia (Arkhangelsk, Leningrad, Voronezh, Yakutia), Sweden. Euro-Siberian temperate. New for Yakutia and eastern Palaearctic.

\section{Campsicnemus armatus (Zetterstedt, 1849)}

REFERENCES. Negrobov, Zlobin, 1978: 51 (C Yakutia: Lena River valley), Negrobov, Chalaya, 1991: 65 (C Yakutia: Lena River valley, Amga River valley, Lena-Amga interfluve); Grichanov, Bagachanova, 2006: 128 (C Yakutia: Amga River valley).

MATERIAL EXAMINED. NE Yakutia: $10^{7}$, Env. vil. Tomtor,

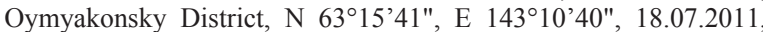
Bagachanova (IBPC); $50^{7}$, Cherskii, 18.08.1971, Gorodkov (ZIN). C Yakutia: $50^{2}+$, Yakutsk, 10.08 .1987 , Zlobin (ZIN); $20^{7}$, Amginsky District, Mikhailovka, 5.08.1987, Zlobin (ZIN). W Yakutia: 20", Leninsky [=Nyurbinsky] District, Nyurba, 28.06.1987, Zlobin (ZIN)

DISTRIBUTION. Type locality: Denmark: Rosenthal, Gryphium. Palaearctic: Austria, Belgium, Czech Republic, Denmark, Finland, France, Germany, Iceland, Iran, Ireland, Italy, Mongolia, Netherlands, Norway, Poland, Russia (Arkhangelsk, Kamchatka, Karelia, Krasnoyarsk, Murmansk, Nenetsia, Tatarstan, Yakutia, Yamal, Yekaterinburg), Slovakia, Sweden, Turkey (Kars), UK, North Africa; Tropical Africa. Multiregional polyzonal. New for NE and W Yakutia.

HABITATS. Banks of rivers and rivulets, swamp meadow, willow bed, Alopecurus at slopes.

\section{Campsicnemus articulatellus (Zetterstedt, 1843)*}

MATERIAL EXAMINED. C Yakutia: Lena-Vilyui interfluve: $10^{7}$, Ulakhan Chabydai Lake, $30 \mathrm{~km} \mathrm{~N}$ vil. Magarassy, 4.09.2012, A. Popov (IBPC).
DISTRIBUTION. Type locality: Sweden: Gottlandia; Oja. Palaearctic: Denmark, Estonia, Finland, France, Germany, Ireland, N Kazakhstan, Latvia, Poland, Russia (Karelia, Leningrad, Pskov, Ryazan, Yakutia); Sweden. Euro-Siberian temperate. New for Yakutia; easternmost distribution.

HABITATS. Cereal grasses at lake.

\section{Campsicnemus bagachanovae Grichanov et Volfov, 2009}

REFERENCES. Grichanov, Volfov, 2009: 964 (C Yakutia: Lena-Amga interfluve).

DISTRIBUTION. Type locality: Central Yakutia, LenaAmga interfluve, Tyungyulyu vil., $50 \mathrm{~km}$ ENE Yakutsk. Conditional endemic of boreal Yakutia.

HABITATS. Hygrophyte communities.

\section{Campsicnemus compeditus Loew, 1857}

REFERENCES. Negrobov, Zlobin, 1978: 52 (C Yakutia: Amga River valley); Negrobov, Chalaya, 1991: 65 (C Yakutia: LenaAmga interfluves).

DISTRIBUTION. Type locality: Poland: "aus hiesiger Gegend" [= Meseritz]. Palaearctic: Austria, Belgium, Czech, Finland, France, Germany, Ireland, Japan, Kyrgyzstan, Latvia, Netherlands, Norway, Poland, Russia (Karelia, Leningrad, Murmansk, Buryatia, S Kamchatka, Yakutia), Sweden, UK, Switzerland. Trans-Eurasian temperate.

\section{Campsicnemus magius (Loew, 1845)}

REFERENCES. Negrobov, Chalaya, 1991: 65 (C Yakutia: Lena-Amga interfluve)

DISTRIBUTION. Type locality: Italy: Sicily. Palaearctic: Algeria, Austria, Azerbaijan, Belgium, Bosnia and Herzegovina, Bulgaria, Czech, France, Germany, Hungary, Iran, Israel, Italy, Netherlands, Spain, Romania, Russia (Astrakhan, Ekaterinburg, Kabardino-Balkaria, Krasnodar, Rostov, Yakutia), Tajikistan, Turkey (Korucuk), Turkmenistan, UK, Ukraine (Odessa), Uzbekistan. Euro-Siberian-CentralAsian, polyzonal.

\section{Campsicnemus paradoxus (Wahlberg, 1844)}

REFERENCES. Negrobov, Zlobin, 1978: 52 (C Yakutia, Aldan River basin).

DISTRIBUTION. Type locality: Sweden: Quickjock. Palaearctic: Finland, Mongolia, Norway, Russia (Murmansk, Yakutia), Sweden. Euro-Siberian, boreal.

\section{Campsicnemus picticornis (Zetterstedt, 1843)}

REFERENCES. Negrobov, Zlobin, 1978: 53 (C Yakutia: Lena River valley); Negrobov, Chalaya, 1991: 66 (C Yakutia: LenaAmga interfluve, Lena River valley; Grichanov, Bagachanova, 2006: 129 (C Yakutia: Lena-Amga interfluve).

MATERIAL EXAMINED. NE Yakutia. $50^{7}+$, Cherskii, 18.08.1971, Gorodkov (ZIN). W Yakutia: $30^{7}+$, Leninsky [=Nyurbinsky] District, Nyurba, 23.06, 30.06.1987, Zlobin (ZIN). C Yakutia: Lena River valley, $60^{7}+$, Yakutsk, 10.08.1987, Zlobin (ZIN); 10', 7 km SW Yakutsk, Plemkhoz, 14.06.2007, Bagachanova (IBPC); $10^{7}$, Pokrovskii road, $39 \mathrm{~km}$ SW Yakutsk, 23.08.2007, Ermakova (IBPC); $10^{7}, 55 \mathrm{~km}$ SW Yakutsk, 23.08.2007, Bagachanova (IBPC); 107, Botanical Garden, 10.08.2005, Evdokarova (IBPC); $10^{\top}, 25$ km N Yakutsk, vil. Kildyamtsy, 4.07.2007, Bagachanova (IBPC); Amga River valley, $10^{7}$, Amginsky District, Mikhailovka, 5.08.1987, Zlobin (ZIN); SW Yakutia: 107, Kyatchi, $21 \mathrm{~km}$ W Olekminsk, 21.07.2008, Bagachanova (IBPC).

DISTRIBUTION. Type locality: Sweden: "Suecia meridionali et media, in Scania ad Raften; Ostrogothia ad Larketorp; Haradshammar; Holmiam". Palaearctic: Asia (Israel, Kazakhstan, Kyrgyzstan, Turkey, Uzbekistan, Russia: Birobidzhan, Blagoveshchensk, Buryatia, Kamchatka, 
Khabarovsk, Krasnoyarsk, Novosibirsk, Sayan Mts., Yakutia, Vladivostok), Europe (Austria, Belgium, Czech Republic, Denmark, Estonia, Finland, France, Germany, Hungary, Italy, Latvia, Netherlands, Poland, Russia (Kaliningrad, Karelia, Leningrad), Slovakia, Sweden, Turkey (Hakkari), UK, Ukraine, Uzbekistan). Trans-Palaearctic temperate. New for NE, W and SW Yakutia.

HABITATS. Artemisia, Stipa, Agropyron and Artemisiacereal communities on steppe slopes; Carex hygrophyte community; shrunken swamp on steppe slope; bank of swampy lake; Carex swamp at rivulet bank; swamp meadow, willow bed.

\section{Campsicnemus pumilio (Zetterstedt, 1843)}

Campsicnemus pectinulatus Loew, 1864.

REFERENCES. Negrobov, Zlobin, 1978: 52 (as Campsicnemus pectinulatus Loew, 1864; C Yakutia: Amga River valley). Negrobov, Chalaya, 1991: 65 (as Campsicnemus pectinulatus Loew, 1864; C Yakutia: Lena River valley).

DISTRIBUTION. Type locality: Sweden: "Sueciam in Scania ad Scandhammar et Silfakra". Palaearctic: Austria, Belgium, Czech, Denmark, Estonia, Finland, France, Germany, N Kazakhstan, Kyrgyzstan, Netherlands, Poland, Romania, Russia (Karelia, Krasnodar, Krasnoyarsk, Leningrad, Moscow, Novgorod, Ryazan, Ulyanovsk, Yakutia, Kamchatka), Sweden, UK. Trans-Eurasian temperate.

\section{Chrysotus andrei Negrobov, 1986a}

REFERENCES. Negrobov, 1986a: 36; Negrobov, 1986b: 11 (as Chrysotus andreji) (C Yakutia: Lena-Amga interfluve).

MATERIAL EXAMINED. C Yakutia: 10", Amginsky District, Mikhailovka, 25.07.1987, Zlobin (ZIN); W Yakutia: 10', vil. Arylakh SE Mirnyi, 8.07.1987, Zlobin (ZIN).

DISTRIBUTION. Type locality: Amur Region, Zeya. Palaearctic: Russia (Blagoveshchensk, Krasnoyarsk, Yakutia), Mongolia. East-Siberian, boreal. New for W Yakutia.

\section{Chrysotus cilipes Meigen, 1824}

REFERENCES. Maslova et al., 2011: 465 (C Yakutia: Amga River valley).

DISTRIBUTION. Type locality: Germany: Hamburg. Palaearctic: Abkhazia, Armenia, Austria, Azerbaijan, Belgium, Bosnia and Herzegovina, Bulgaria, China, Czech Republic, Estonia, Denmark, Finland, France, Germany, Hungary, Ireland, Italy, Japan, Kazakhstan, Korea, Latvia, Lithuania, Luxembourg, Mongolia, Netherlands, Norway, Poland, Portugal (Madeira), Romania, Russia (Adygea, Altai Rep., Altai Terr., Baikal, Blagoveshchensk, Buryatia, Chita, Irkutsk, KabardinoBalkaria, Karachai-Cherkessia, Karelia, Khabarovsk, Krasnodar, Krasnoyarsk, Moscow, Leningrad, Novgorod, Novosibirsk, Pskov, Rostov, Sakhalin, Tomsk, Vladivostok, Voronezh, Yakutia), Slovakia, Spain, Sweden, Switzerland, Turkey, UK, Ukraine. Trans-Palaearctic, polyzonal.

\section{Chrysotus degener Frey, 1917}

REFERENCES. Maslova, 2006: 106 (C Yakutia: Lena River valley; unpubl.); Grichanov, 2017: 131.

DISTRIBUTION. Type locality: Sri Lanka: Anuradhapura. Palaearctic: Russia (Blagoveshchensk, Vladivostok, Yakutia); Oriental: China (Taiwan), India, Myanmar, Sri Lanka, Pakistan. Multiregional polyzonal.

\section{Chrysotus femoratus Zetterstedt, 1843}

REFERENCES. Negrobov, Chalaya, 1991: 66 (C Yakutia: Lena River valley, Lena-Amga interfluve; Lena-Vilyui interfluve).

DISTRIBUTION. Type locality: Denmark: Amager. Palaearctic: Austria, Belgium, China, Czech Republic, Denmark, Estonia, Finland, France, Georgia,Germany, Greece, Hungary, Ireland, Italy, Latvia, Lithuania, Moldova, Mongo- lia, Netherlands, Norway, Poland,Portugal (Madeira), Romania, Russia (Adygea, Altai, Arkhangelsk, Blagoveshchensk, Irkutsk, Kamchatka, Karelia,Karachai-Cherkessia, Khabarovsk, Krasnodar, Leningrad, Murmansk, Novgorod, Pskov, Ryazan, Sakhalin,Tatarstan, Vladivostok, Voronezh, Yakutia), Slovakia, Sweden, Switzerland, Turkey (Erzurum, Hakkari), UK, Ukraine. Trans-Palaearctic, polyzonal.

14. Chrysotus fuscoluteus Negrobov et Zurikov, 1986 REFERENCES. Negrobov, Tsurikov, 1986: 632 (N Yakutia and NW Yakutia).

DISTRIBUTION. Type locality: Russia: Yakutia, lower Lena River, Ayakit. Palaearctic: Russia (Yakutia). Conditional endemic, Arctic-boreal.

\section{Chrysotus glebi Negrobov et Maslova, 1995}

REFERENCES. Maslova, 2006: 109 (C Yakutia: Lena-Amga interfluve; unpubl.); Grichanov, 2017: 133.

DISTRIBUTION. Type locality: Russia: Primorye, Ussuri Reserve. Palaearctic: Kyrgyzstan, Russia (Altai, Blagoveshchensk, Karachai-Cherkessia, Leningrad, Murmansk, Vladivostok, Yakutia), Spain, Turkey (Rize), Ukraine. Trans-Eurasian temperate.

\section{Chrysotus gramineus (Fallén, 1823)}

REFERENCES. Negrobov, Chalaya, 1991: 67 (C Yakutia: Lena River valley, Lena-Amga interfluve, Amga River valley; N Yakutia; NE Yakutia).

DISTRIBUTION. Type locality: not given [Sweden]. Palaearctic: Abkhazia, Andorra, Armenia, Austria, Azerbaijan, Belarus, Belgium, Bosnia and Herzegovina, Bulgaria, China; Czech Republic, Denmark, Estonia; Finland, France, Georgia, Germany, Greece, Hungary, Ireland, Italy, Korea, Kyrgyzstan, Latvia, Lithuania, Moldova, Netherlands, Norway, Poland, Romania, Russia (Adygea, Alania, Altai Rep., Arkhangelsk, Bashkortostan, Blagoveshchensk, Buryatia, Chelyabinsk, Chita, Chukotka, Crimea, Irkutsk, KabardinoBalkaria, Karachai-Cherkessia, Karelia, Khabarovsk, Kostroma, Krasnodar, Krasnoyarsk, Kursk, Leningrad, Lipetsk, Mordovia, Moscow, Murmansk, Novgorod, Orel, Orenburg, Pskov, Ryazan, Sakhalin, Tatarstan, Tomsk, ?Ural, Vologda, Voronezh, Yakutia, Yaroslavl), Slovakia, ?Slovenia, Spain, Sweden, Switzerland, Tajikistan, Turkey (Hakkari, Erzurum, Kars), Turkmenistan, UK, Ukraine (Cherkasy, Kharkiv, Kherson). Multiregional polyzonal.

\section{Chrysotus laesus (Wiedemann, 1817)}

REFERENCES. Maslova et al., 2011: 466 (NE Yakutia).

DISTRIBUTION. Type locality: Germany: Kiel. Palaearctic: Armenia, Austria, Belarus, Belgium, Bosnia and Herzegovina, Bulgaria, China, Czech Republic, Denmark, Estonia, Finland, France, Georgia, Germany, Hungary, Italy, N Kazakhstan, Latvia, Lithuania, Moldova, Netherlands, Norway, Poland, Romania, Russia (Adygea, Altai Rep., Bashkortostan, Blagoveshchensk, Buryatia, Chechnya, Chelyabinsk, Crimea, Dagestan, Ekaterinburg, Irkutsk, Karachai-Cherkessia, Karelia, Krasnodar, Krasnoyarsk, Kursk, Leningrad, Lipetsk, Magadan, Moscow, Orenburg, Omsk, Pskov, Ryazan, Tatarstan, Tomsk, Vladivostok, Voronezh, Yakutia, Yaroslavl), Serbia, Slovakia, Slovenia, Spain, Sweden, Switzerland, Tajikistan, Turkey (Kars), UK, Ukraine (Carpathiens, Cherkasy, Kharkiv). Trans-Palaearctic temperate.

\section{Chrysotus ljutengensis Negrobov et Zurikov,} 1986

REFERENCES. Negrobov, Tsurikov, 1986: 632 (C Yakutia: Lena-Amga interfluve). 
MATERIAL EXAMINED. W Yakutia: $10^{7}$, Leninsky [=Nyurbinsky] District, Nyurba, 28.06.1987, Zlobin (ZIN); SW Yakutia: $20^{7}$, Lensky District, vil. Tolon, 16.07.1987, Zlobin (ZIN)

DISTRIBUTION. Type locality: Russia: Yakutia, Lyutenge River, road to Amga River. Palaearctic: Russia (Yakutia). Conditional endemic, boreal. New for W and SW Yakutia.

19. Chrysotus neglectus (Wiedemann, 1817)

REFERENCES. Negrobov, Chalaya, 1991: 68 (C Yakutia: Lena-Amga interfluve; Amga River valley; Lena River valley).

MATERIAL EXAMINED. C Yakutia: 207', Yakutsk, 19 20.07.1987, Zlobin (ZIN).

DISTRIBUTION. Type locality: "Holstein". Palaearctic: Armenia, Austria, Belarus, Belgium, Bosnia and Herzegovina, China (Shanghai) Czech, Denmark, Estonia, Germany, Ireland, Finland, France, Hungary, Kazakhstan, Kyrgyzstan, Latvia, Lithuania, Madeira, Mongolia, Netherlands, Norway, Poland, Romania, Russia (Alania, Altai, Arkhangelsk, Blagoveshchensk, Bryansk, Buryatia, Chechnya, Chelyabinsk, Chita, Dagestan, Kamchatka, Karelia, Khabarovsk, Krasnodar, Krasnoyarsk, Kursk, Leningrad, Mordovia, Moscow, Murmansk, Novgorod, Omsk, Pskov, Samara, Tomsk, Vladivostok, Voronezh, Yakutia), Spain, Sweden, Switzerland, Tajikistan, UK, Ukraine (Cherkasy, Kiev, Lvov, Sumy, Uzhgorod), Uzbekistan. Trans-Palaearctic polyzonal.

\section{Chrysotus nudisetus Negrobov et Maslova, 1995}

REFERENCES. Negrobov, Maslova, 1995: 460 (NE Yakutia). DISTRIBUTION. Type locality: Russia: Sakhalin, Anivskii distr. Palaearctic: Japan, Russia (Chukotka, Khabarovsk, Magadan, Sakhalin, Vladivostok, Yakutia). East-SiberianFar-Eastern temperate.

\section{Chrysotus obscuripes Zetterstedt, 1838}

REFERENCES. Grichanov, 2002: 122 (C Yakutia: Lena-Amga interfluve)

DISTRIBUTION. Type locality: Sweden: "Lapponica Umensi, Fredrica". Palaearctic: Armenia, Austria, Belgium, China, Czech Republic, Denmark, Estonia, Finland, France, Germany, Ireland, Kyrgyzstan, Latvia, Lithuania, Netherlands, Norway, Romania, Russia (Blagoveshchensk, Karelia, Leningrad, Yakutia), Sweden, Switzerland, Turkey (Erzurum, Kars), UK, Ukraine. Euro-Siberian-Central-Asian, boreal-montane

\section{Chrysotus orientalis Negrobov et Zurikov, 2000}

REFERENCES. Negrobov et al., 2000b: 231 (C Yakutia: Lena River valley, Lena-Amga interfluve).

MATERIAL EXAMINED. C Yakutia: 10', Yakutsk, Botanical Garden, 20.07.1987, Zlobin (ZIN)

DISTRIBUTION. Type locality: [Inner] Mongolia: Khent. aimak, Kerulen River, 40 km WSW Bayan-Obo. Palaearctic: China (Inner Mongolia), Mongolia, Russia (Altai Rep., Buryatia, Chita, Vladivostok, Yakutia). East-Siberian-Far-Eastern temperate.

\section{Chrysotus pulchellus Kowarz, 1874}

REFERENCES. Maslova, 2006: 119 (NE Yakutia; unpubl.); Grichanov, 2017: 131

MATERIAL EXAMINED. W Yakutia: $10^{7}$, Suntarsky District, vil. Kutana, 2.07.1987, Zlobin (ZIN); C Yakutia: 607, Amginsky District, Mikhailovka, 24, 25, 27.07.1987, Zlobin (ZIN).

DISTRIBUTION. Type locality: Asch [=Ash, Czech Republic]. Palaearctic: Armenia, Austria, Belgium, Belarus, Bulgaria, China, Czech Republic, Denmark, Estonia, Finland, France, Georgia, Germany, Greece, Hungary, Italy, Kyrgyzstan, Latvia, Lithuania, Luxembourg, Mongolia, Netherlands, Norway, Poland, Romania, Russia (Adygea, Irkutsk,
Karelia, Krasnodar, Krasnoyarsk, Leningrad, Murmansk, Ryazan, Sayan Mountains, Tatarstan, Voronezh, Yakutia), Slovakia, Spain, Sweden, Switzerland, Turkey (Adapazari), UK, Ukraine. Euro-Siberian-Central-Asian, temperate. New species for $\mathrm{C}$ and $\mathrm{W}$ Yakutia.

\section{Chrysotus sibiricus Negrobov et Maslova, 1995*}

MATERIAL EXAMINED. SW Yakutia: $20^{\top}$, Biryuk River, 4 $\mathrm{km}$ from Melichan River mouth, 13-14.07.2008, Bagachanova (ZIN); $10^{7}$, Biryuk River mouth, 11.07.2008, Bagachanova (ZIN).

DISTRIBUTION. Type locality: Siberia: Nizhnyaya Angara, Taltsy. Palaearctic: Russia (Irkutsk, Krasnoyarsk, Kamchatka, Yakutia). East-Siberian-Far-Eastern boreal. New species for Yakutia.

HABITATS. Allium, Carex and Eleocharis communities at river banks.

\section{Chrysotus smithi Negrobov, 1980*}

REFERENCES. Maslova, 2006: 124 (C Yakutia: Amga River valley; unpubl.).

MATERIAL EXAMINED. W Yakutia: 107, Suntarsky District, vil. Kutana, 4.07.1987, Zlobin (ZIN)

DISTRIBUTION. Type locality: Russia: Baikal, Listvyanka, Barguzin Reserve, Davshe. Palaeartic: Mongolia, Russia (Blagoveshchensk, Buryatia, Yakutia). East-SiberianFar-Eastern, boreal. First published record from Yakutia.

\section{Chrysotus suavis Loew, 1857}

REFERENCES. Negrobov, Chalaya, 1991: 69 (C Yakutia: Lena River valley, Amga River valley, Lena-Amga interfluve); Maslova et al., 2011: 467 (NW, C and NE Yakutia).

DISTRIBUTION. Type locality: Germany: "Coln"; Austria: "Neusiedler See in Ungarn". Palaearctic: Afghanistan, Algeria, Armenia, Austria, Azerbaijan, Belgium, Bosnia and Herzegovina, Bulgaria, N China, Croatia, Czech Republic, Egypt, Estonia, Finland, France, Georgia, Germany, Greece (North Aegean), Hungary, Iraq, Iran, Israel, Italy, Kyrgyzstan, Latvia, Lithuania, Middle Asia, Mongolia, Morocco, Netherlands, Norway, Poland, Romania, Russia (Adygea, Alania, Altai Rep., Astrakhan, Blagoveshchensk, Buryatia, Irkutsk, Kabardino-Balkaria, Kamchatka, Khabarovsk, Krasnodar, Krasnoyarsk, Kursk, Leningrad, Lipetsk, Magadan, Pskov, Rostov, Ryazan, Sakhalin, Tatarstan, Voronezh, Yakutia), Serbia, Slovakia, Spain (Canary Is), Sweden, Switzerland, Turkey (Adiyaman, Antalya, Antakya, Artvin, Gaziantep, Kizildere, Sanliurfa), UK, Ukraine (Cherkasy, Kherson, Odessa). Trans-Palaearctic, polyzonal.

HABITATS. Cornus communities on meadows.

\section{Chrysotus viridifemoratus von Roser, 1840}

Chrysotus monochaetus Kowarz, 1874.

REFERENCES. Negrobov, Chalaya, 1999: 68 (as Chrysotus monochaetus Kowarz) (C Yakutia: Lena-Amga interfluve); Negrobov et al., 2000a: 84).

DISTRIBUTION. Type locality: not given [Germany: Wurttemberg]. Palaearctic: Austria, Belgium, Czech, England, France, Germany, Hungary, Italy, Netherlands, Poland, Romania; Russia (Krasnoyarsk Terr., Novosibirsk Region, Taimyr, Yakutia, Yamalia), Slovakia, Switzerland; Ukraine (Carpathiens, Chernovtsy). Euro-Siberian temperate.

\section{Chrysotus zlobiniani Negrobov et Maslova, 1995}

REFERENCES. Maslova, 2006: 127 (NE Yakutia; unpubl.). DISTRIBUTION. Type locality: Russia: Sakhalin, Anivskii distr., Lyutaga River. Palaearctic: Russia (Sakhalin, Yakitia). North-East-Siberian-Far-Eastern boreal. 


\section{Diaphorus nigricans Meigen 1824*}

MATERIAL EXAMINED. SW Yakutia: $2 \sigma^{7}$, Biryuk River, left tributary of Lena River, 14 km from mouth, 16.07.2008, Bagachanova (IBPC).

DISTRIBUTION. Type locality: Germany. Palaearctic: Abkhazia; Austria, Belarus, Belgium, China, Czech, Denmark, Estonia, Finland, France, Germany, Greece, Hungary, India: Kashmir, Ireland, Italy, Israel; Netherlands, Norway, Poland, Romania, Russia (Karelia, Krasnodar, Leningrad, Moscow, Murmansk, Yakutia); Spain, Sweden, Switzerland, UK; Afrotropical, Nearctic, Oriental and Neotropical Regions. Multiregional polyzonal. New for Yakutia.

HABITATS. Plant communities at river bank; Carex communities at spruce forest.

\section{Dolichopus agilis Meigen, 1824}

REFERENCES. Stackelberg, 1930a: 27 (C Yakutia: Yakutsk); Grichanov, Bagachanova, 2006: 130 (C Yakutia: Lena-Amga interfluve).

DISTRIBUTION. Type locality: not given. Palaearctic: Andorra, Austria, Belgium, Bosnia and Herzegovina, Czech, China (Hebei, Inner Mongolia, Ningxia, Gansu), Denmark, Estonia, France, Germany, Hungary, Italy, Kazakhstan, Poland, Netherlands, Slovakia, Sweden, Switzerland, Russia (Altai Rep., Blagoveshchensk, Buryatia, Irkutsk, Kuril Is., Novosibirsk, Sayan Mts., Vladivostok, Yakutia), UK, Ukraine. Trans-Eurasian temperate.

31. Dolichopus amginensis Stackelberg, 1928

REFERENCES. Stackelberg, 1928: 267; Stackelberg, 1930a: 29 (C Yakutia: Lena-Amga interfluve)

DISTRIBUTION. Type locality: Russia: Yakutsk Distr., Abyj, Berdzhanstiach. Palaearctic: Russia (N Ural, Yakutia). Ural-Siberian boreal.

\section{Dolichopus angustipennis Kertesz, 1901}

REFERENCES. Stackelberg, 1930a: 31 (C Yakutia: Yakutsk); Negrobov, Chalaya, 1991: 69 (C Yakutia: Lena-Amga interfluve; Lena River valley); Grichanov, 2002: 122 (C Yakutia: Lena-Amga interfluve); Grichanov, Bagachanova, 2006: 130 (C Yakutia: LenaAmga interfluve, Lena River valley).

MATERIAL EXAMINED. W Yakutia: $30^{7}, 1 \circ$, Kyukei Lake, vil. Tumul env., $60 \mathrm{~km} \mathrm{NE}$ vil. Suntar, N 6243'38", E $117^{\circ} 45^{\prime} 44^{\prime \prime}$, 27.06.2011, Sivtseva (IBPC); $10^{7}$, Sordonnokh Lake, $3 \mathrm{~km}$ NW vil. Kempendyai, N 6202'57", E 118 36’03", 24.04.2011, Sivtseva (IBPC); C Yakutia: 107, Khomustakh, 27.07.1969, Sychevskaya (ZIN); Lena-Amga interfluve: 107, 3, 45 km NE Yakutsk, Kysynny Aryylaakh, 19.06.2008, Bagachanova (IBPC); 10², 1, vil. Borgontsy env., $95 \mathrm{~km}$ NE Yakutsk, 19.06.2008, Bagachanova (IBPC); Lena River valley: 2 , , Khatassy, $10 \mathrm{~km} \mathrm{SW}$ Yakutsk, pig farm, 16.07.1986, Bagachanova (IBPC); Amga River valley: 10, 1 , Amga River, Mikhailovka, 22.06.1986, Bagachanova (IBPC); SW Yakutia: 1ㅇ, Biryuk River mouth, 11.07.2008, Bagachanova (IBPC)

DISTRIBUTION. Type locality: Russia: Kazan. Palaearctic: Finland, Germany, Russia (Buryatia, Irkutsk, Kamchatka, Karelia, Tatarstan, Primorskii Terr., Yakutia), Georgia, Kazakhstan, China. Trans-Palaearctic boreal-montane. New for W and SW Yakutia.

HABITATS. Cereals; Carex, Scolochloa, Eleochara mesophyte and hygrophyte communities; lake banks.

\section{Dolichopus annulipes Zetterstedt, 1838}

REMARKS. We didn't find publications with the original material for this species collected eastward of the Urals. Most probably, Yakutia, Magadan Region and Primorsky Territory were in error included in the species area $[\mathrm{Ne}-$ grobov et al., 2000a: 84], which was repeated in a number of the subsequent publications.

\section{Dolichopus annulitarsis Ringdahl, 1920}

REFERENCES. Stackelberg, 1930a: 31 (NW Yakutia); Grichanov, Bagachanova, 2006: 130 (C Yakutia: Lena River valley).

MATERIAL EXAMINED. W Yakutia: $10^{7}$, vil. Arylakh, SE Mirnyi, 8, 9.07.1987, Zlobin (ZIN).

DISTRIBUTION. Type locality: Sweden: Lappland, Tornetrask. Palaearctic: Sweden, Russia (Karelia, N Ural, Yakutia, Magadan, Kamchatka); Nearctic: Alaska. Holarctic, Arctic-boreal. New for W Yakutia.

\section{Dolichopus basalis Loew, 1859*}

MATERIAL EXAMINED. C Yakutia: Lena-Viluy interfluve: $10^{7}$, Keptik, 20 km NW Berdigestyakh, 10.07.1982, Bagachanova (ZIN).

DISTRIBUTION. Type locality: "Chamar-Daban" Ridge (Siberia). Palearctic: Korea, Russia (Buryatia, Chita, Irkutsk, Kamchatka, Khabarovsk, Yakutia). East-Siberian-Far-Eastern, temperate. New for Yakutia.

HABITATS. Spiraea salicifolia communities.

\section{Dolichopus bianchii Stackelberg, 1929}

REFERENCES. Stackelberg, 1929: 173; 1930: 36 (C Yakutia: Lena-Amga interfluve); Negrobov, Chalaya, 1991: 70 (C Yakutia: Lena River valley); Grichanov, 2002: 122 (C Yakutia: Lena-Amga interfluve); Grichanov, Bagachanova, 2006: 130 (C Yakutia: LenaAmga interfluve).

MATERIAL EXAMINED. SW Yakutia: 10', Biryuk River mouth, 11.07.2008, Bagachanova (IBPC).

DISTRIBUTION. Type locality: Russia: "Nord Ural: Voikar-Flufisysteme; Jakutien: Keedej-See, Berdzhastjach". Palaearctic: China, Mongolia, Russia (Yamalia, Yakutia, Khabarovsk, Vladivostok). Siberian-Far-Eastern temperate. New for SW Yakutia.

HABITATS. Riparian communities, horsetail, spruce.

\section{Dolichopus brevipennis Meigen, 1824}

REFERENCES. Stackelberg, 1930a: 39 (C Yakutia); Negrobov, Chalaya, 1991: 70 (C Yakutia: Lena River valley, Lena-Amga interfluve, Amga River valley); Grichanov, 2002: 122; Grichanov, Bagachanova, 2006: 130 (C Yakutia: Lena-Amga interfluve, Lena River valley; SW Yakutia).

MATERIAL EXAMINED. W Yakutia: Kyukei Lake, vil. Tumul env., 60 km N vil. Suntar, N 6243'38", E 11745'44", 27.06.2011, Sivtseva (IBPC); C Yakutia: Lena River valley: $20^{7}$, Yakutsk, 19.07.1987, Zlobin (ZIN); 10', Elanka, alas Ebe, , 30.06.2008, Bagachanova (IBPC); SW Yakutia: 10', Biryuk River mouth, 11.07.2008, Bagachanova (IBPC); 1요 env. vil. Solyanka, env. Olekminsk, 25.08.2008, Bagachanova (IBPC).

DISTRIBUTION. Type locality: Sweden. Palaearctic: Austria, Belarus, Belgium, China (Xinjiang), Czech Republic, Denmark, Estonia, Finland, France, Germany, Ireland, Italy, N Kazakhstan, Latvia, Lithuania, Netherlands, Norway, Poland, Russia (Adygea, Altai Rep., Altai Terr., Arkhangelsk, Baskortostan, Buryatia, Cis-Baikalia, Irkutsk, Kabardino-Balkaria, Karelia, Khantia-Mansia, Krasnodar, Kirov, Komi, Leningrad, Moscow, Murmansk, Novgorod, Omsk, Orenburg, Perm, Pskov, Ryazan, Tomsk, Tyumen, Voronezh, Yaroslavl, Vladivostok, Yakutia), Sweden, UK; Nearctic: Canada and USA: Alaska, Yukon, British Columbia, Northwest Terr., Montana, Alberta, Saskatchewan, Quebec, Prince Edward Is, Newfoundland (Labrador). Holarctic temperate. New for W Yakutia.

HABITATS. Scolochloa, Carex, Rumex, Alisma, cereal hygrophyte communities on meadows, river banks, swamps, spruce forest edge.

\section{Dolichopus calceatus Parent, 1927}

REFERENCES. Grichanov, 2002: 122 (C Yakutia: Lena-Amga 
interfluve); Grichanov, Bagachanova, 2006: 130 (C Yakutia: LenaAmga interfluve, Lena River valley).

MATERIAL EXAMINED. C Yakutia: 10', env. vil. Markha 10 km N Yakutsk, ISL station, 19.07.2004, Bagachanova (ZIN).

DISTRIBUTION. Type locality: Russia: "Transbaikale; Pjetschanka, b. Tschita". Palaearctic: China, Kazakhstan, Russia (Tomsk, Chita, Irkutsk, Amur, Kamchatka Regions, Altai Rep., Khabarovsk, Primorskii Terr., Buryatia, Yakutia). Siberian-Far-Eastern temperate.

HABITATS. Riparian plant communities.

\section{Dolichopus clavipes Haliday, 1832}

REFERENCES. Stackelberg, 1930a: 44 (C Yakutia: Yakutsk); Negrobov, Chalaya, 1991: 70 (C Yakutia: Lena River valley); Negrobov, Rodionova, 2004: 192 (C Yakutia: Yakutsk); Grichanov, 2002: 122 (C Yakutia: Lena-Amga interfluve); Grichanov, Bagachanova, 2006: 131 (C Yakutia: Lena-Amga interfluve, Lena River valley).

MATERIAL EXAMINED. C Yakutia: Lena-Amga interfluve: $2 \sigma^{7}$, Borogontsy, $96 \mathrm{~km}$ NE Yakutsk, alas, 19.06.2008, Bagachanova (IBPC); 39, Tyungyulyu, Kysynny Aryylaakh, Bagachanova (IBPC); 19, Balyktakh, $55 \mathrm{~km} \mathrm{~N}$ Yakutsk, alas Ilin Keeser, 19.07.2008, Bagachanova (IBPC); Lena River valley: 10 , 19, 37 $\mathrm{km} \mathrm{N}$ vil. Namtsy, 24.06.2011, Nogovitsyna (IBPC); $3 \sigma^{7}, 2$, Elanka, alas Ebe, 30.06.2008; 1오. Elanka, Lena River bank, 28.08.2008; 5ㅇ, Oktemtsy, 12, 17, 23.06.1977; 1우웅, Khatassy, pig farm, 16.07.2006; 2 9 , Breeding farm, $5 \mathrm{~km}$ SW Yakutsk, 14.06.2006, Bagachanova (IBPC); $1 \sigma^{7}$, Yakutsk, 21.07.1987, Zlobin $(\mathrm{ZIN})$; SW Yakutia: $1+$, $1 \sigma^{\top}$, Biryuk River mouth, 11.07.2008, Bagachanova (IBPC); W Yakutia: 6 $0^{7}$, Leninsky [=Nyurbinsky] District, Nyurba, 25, 30.06.1987, Zlobin (ZIN); 20', Suntarsky District, vil. Kutana, 2.07.1987, Zlobin (ZIN)

DISTRIBUTION. Type locality: Ireland: Holywood. Palaearctic: Austria, Belgium, Czech, China (Xinjiang), Denmark, Finland, France, Germany, Hungary, Iran, Ireland, Kazakhstan, Mongolia, Netherlands, Norway, Poland, Russia (Buryatia, Irkutsk, Krasnodar, Krasnoyarsk, Murmansk, Novosibirsk, Orenburg, Yakutia), Spain, Sweden, Tajikistan, UK, Ukraine (Kherson, Odessa, Zaporizhzhya), Uzbekistan. Euro-Siberian-Central-Asian temperate. New for W and SW Yakutia.

HABITATS. Riparian and forb-cereal plant communities; hygrophyte, mesophyte, xerophyte communities; lake banks, steppe slopes, meadows, swamps; willow bed, birch forest, Potentilla, Equisetum.

REMARKS. Stackelberg [1930a: 45] and Negrobov, Chalaya [1991: 70] recorded also subspecies Dolichopus clavipes fusiformis Becker, 1917 from the Republic (C Yakutia: Lena-Amga interfluve; Lena River valley).

\section{Dolichopus costalis Frey, 1915}

REFERENCES. Stackelberg, 1930a: 45 (C Yakutia: Yakutsk); Negrobov, Chalaya, 1991: 70 (C Yakutia: Lena River valley; LenaAmga interfluve).

DISTRIBUTION. Type locality: Russia: "Petrosawodsk". Palaearctic: Finland, Sweden, Russia (Karelia, Yakutia), Mongolia. Euro-Siberian boreal.

\section{Dolichopus czekanovskii Stackelberg, 1928}

REFERENCES. Stackelberg, 1928: 263; Stackelberg, 1930a: 47 (NW Yakutia).

DISTRIBUTION. Type locality: Russia: "Nord-Sibirien, Olenyok". Palaearctic: Russia (Altai Rep., Yakutia); Nearctic: Canada, USA. East-Siberian-Nearctic, Arctic-boreal.

\section{Dolichopus discifer Stannius, 1831}

Dolichopus nigricornis Becker, 1917 et auctt. (nec Meigen, 1824), misidentification.

REFERENCES. Negrobov et al., 2000: 84 (as Dolichopus nigricornis Meigen, 1824).
MATERIAL EXAMINED. S Yakutia: $10^{7}$, Gorkit, $80 \mathrm{~km}$ from vil. Torgo, point 4, 8.07.2010, Potapova (IBPC).

DISTRIBUTION. Type locality: Germany. Palaearctic: Austria, Belgium, Belarus (Minsk), Bulgaria, Czech, Denmark, England, Estonia, Finland, France, Georgia, Germany, Hungary, Ireland, Italy, N Kazakhstan, Latvia, Lithuania, Netherlands, Norway, Poland, Romania, Russia (Altai Rep., Baikal, Karelia, Khabarovsk, Leningrad, Mordovia, Moscow, Murmansk, Novgorod, Sakhalin, Vladivostok, Vologda, Yakutia), Slovakia, Sweden, Switzerland, Ukraine (Kharkiv); Nearctic: Canada, USA: Alaska, British Columbia to Quebec and Nova Scotia, southward to Colorado and New York. Holarctic temperate. First reliable record from Yakutia.

\section{Dolichopus divisus Becker, 1917*}

MATERIAL EXAMINED. NEz Yakutia: 10', Verkhoyanskii Ridge, Ilderkei Lake, Orto-Sala river head, 970 m a. s. 1., 18.07.1991, Vinokurova (ZIN).

DISTRIBUTION. Type locality: Russia: "Urga, Irkutsk". Palaearctic: China, Mongolia, Russia (Irkutsk, Yakutia). New for Yakutia.

\section{Dolichopus eous Stackelberg, 1929}

REFERENCES. Stackelberg, 1930a: 50 (C Yakutia: Yakutsk).

DISTRIBUTION. Type locality: Russia: "Flus Ona and Jakutsk". Palaearctic: Mongolia, Russia (Yakutia, Baikal, Vladivostok). East-Siberian-Far-Eastern temperate.

\section{Dolichopus flavipes Stannius, 1831}

REFERENCES. Stackelberg, 1930a: 53 (C Yakutia: Yakutsk). Negrobov, Chalaya, 1991: 70 (C Yakutia: Lena River valley).

DISTRIBUTION. Type locality: France: Marseille. Palaearctic: Austria, ?Denmark, Finland, France, Germany, Czech, Hungary, Poland, Romania, Russia (Irkutsk, Buryatia, Krasnoyarsk, Yakutia, Russian Far East), ?Sweden, Switzerland, Uzbekistan; Nearctic: USA: Alaska. Holarctic temperate.

\section{Dolichopus fraterculus Zetterstedt, 1843}

REFERENCES. Grichanov, Bagachanova, 2006: 131 (NE Yakutia, NW Yakutia).

MATERIAL EXAMINED. NE Yakutia: $10^{\top}, 19,15 \mathrm{~km}$ NE vil. Khaiysardakh Khaiysardakh, 18.07.2010, Potapova (IBPC); SW Yakutia: $10^{7}$, Biryuk River, $5 \mathrm{~km}$ from Melichan River mouth, 14.07.2008, Bagachanova (IBPC); S Yakutia: 10 $97 \mathrm{~km} \mathrm{~W}$ vil. Chulman, 10.07.2010, Potapova (IBPC).

DISTRIBUTION. Type locality: Sweden: Jemtlandia meridionali, Asarne. Palaearctic: Finland, Lithuania, Norway, Sweden, Russia (Altai Rep., Karelia, Murmansk, Yamalia, Yakutia, Magadan, Kuril Is.); Nearctic: Canada, USA. Holarctic boreal. New for SW and S Yakutia.

HABITATS. Hummocky tundra, birch-sedge communities.

\section{Dolichopus galeatus Loew, 1871}

REFERENCES. Stackelberg, 1930a: 55 (C Yakutia: Yakutsk); Negrobov, Chalaya, 1991: 71 (C Yakutia: Yakutsk); Grichanov, 2002: 123 (C Yakutia: Lena-Amga interfluve); Grichanov, Bagachanova, 2006: 130 (C Yakutia: Lena-Amga interfluve, Lena River valley).

MATERIAL EXAMINED. NE Yakutia: 1, Verkhoyanskii Ridge, Ilderkei Lake, Orto-Sala river head, 21.07.1991, $750 \mathrm{~m}$ a. s. 1., 18.07.1991, Vinokurov (IBPC); C Yakutia: Lena River valley: $10^{7}$, Yakutsk, 18.07.1969, Sychevskaya (ZIN); 107, Khomustakh, 27.07.1969, Sychevskaya (ZIN); 19, Oktemtsy, 28.06.1977, Bagachanova (IBPC); 1 ㅇ․ Tabaginskii cape, $36 \mathrm{~km}$ SW Yakutsk, Malaise trap, 28-30.06.2009, Bagachanova (IBPC); 10 $0^{7}, 2$, Labyda Lake, $17 \mathrm{~km}$ SW vil. Sitte, 21.07.2012, Sivtseva (IBPC); Amga River valley: $20^{7}, 2$ ㅇ․ Amginsky District, Mikhailovka, 24, 28.07, 2.08.1987, Zlobin (ZIN). 
DISTRIBUTION. Type locality: Russia: "Sibirien". Palaearctic: (Blagoveshchensk, Buryatia, Irkutsk, Kamchatka, Khabarovsk, Yakutia), China (Heilongjiang). East-SiberianFar-Eastern temperate. New for NE Yakutia.

HABITATS. Hummocky tundra, birch-sedge and cerealsedge hygrophyte communities.

47. Dolichopus gorodkovi Negrobov, 1973

REFERENCES. Negrobov, Chalaya, 1991: 70 (C Yakutia: Yakutsk); Grichanov, Bagachanova, 2006: 130 (W Yakutia: C Yakutia: Lena River valley).

MATERIAL EXAMINED. C Yakutia: $20^{7}+$, Amga River valley: vil. Megino-Aldan, $20 \mathrm{~km}$ downstream Amga mouth, floodplain, 30.06.1981, Bagachanova (IBPC); 10 , Middle Lena River, $10 \mathrm{~km}$ upsttream Aldan River mouth, 2.07.2002, Potapova N.K. (ZIN)

DISTRIBUTION. Type locality: Russia: Sayan Mountains, Khargun, 6 km E Kyren. Palaearctic: Russia (Buryatia, Chita, Irkutsk, Vladivostok, Yakutia). East-Siberian-Far-Eastern temperate.

\section{Dolichopus griseifacies Becker, 1917*}

MATERIAL EXAMINED. SW Yakutia: 10', Biryuk River, 5 $\mathrm{km}$ from Melichan River mouth, 14. 07.2008, Bagachanova (IBPC) C Yakutia: $10^{7}$, Lena-Vilyui interfluve, Baranattalakh Lake, $12 \mathrm{~km}$ SE vil. Mastakh, 9.08.2012, Potapova N.K. (ZIN).

DISTRIBUTION. Type locality: Russia: "Irkutsk, Sibirien". Palaearctic: Russia (Blagoveshchensk, Buryatia, Irkutsk, Khabarovsk, Vladivostok, Yakutia). East-Siberian-Far-Eastern temperate. New for Yakutia.

HABITATS. Riparian plant communities; Calamagrostis, Carex.

\section{Dolichopus humilis Van Duzee, 1921}

REFERENCES. Negrobov, Grichanov, 2013: 499 (N Yakutia).

DISTRIBUTION. Type locality: USA: Alaska-Yukon Territory boundary. Palaearctic: Russia (Chukotka, Taimyr, N Yakutia, Yamalia); Nearctic: USA (Alaska), Canada, Greenland. Holarctic Arctic.

50. Dolichopus ivanovi Stackelberg, 1929

REFERENCES. Stackelberg, 1929: 170 (C Yakutia: Lena River valley).

DISTRIBUTION. Type locality: Russia: "Kreis Jakutsk: Katshikatskij Nasleg, am rechten Uferungefahr $100 \mathrm{~km}$ aufwarts von Jakutsk". Palaearctic: Russia: Yakutia, Kamchatka; Nearctic: USA: Alaska. East-Siberian-Alaskan boreal.

\section{Dolichopus jacutensis Stackelberg, 1929}

REFERENCES. Stackelberg, 1929: 176; Negrobov, Chalaya, 1991: 71: (C Yakutia: Lena-Amga interfluve, Amga River valley; NE Yakutia; W Yakutia); Grichanov, Bagachanova, 2006: 132 (C Yakutia: Lena River valley).

MATERIAL EXAMINED. S Yakutia: $20^{7}$, Aldan upland, Oyumrak River mouth, 10.08.2010, Nogovitsyna (IBPC).

DISTRIBUTION. Type locality: Russia: "Jakutien, Tumullur-anna, bei Amga, Chattygm-tordo, bei Amga; Werchojansk". Palaearctic: Russia (Yakutia, Magadan, Blagoveshchensk). East-Siberian-Far-Eastern boreal. New for S Yakutia.

52. Dolichopus jakutus Selivanova et Negrobov, 2011

REFERENCES. Selivanova, Negrobov, 2011: 64 (C Yakutia: Lena River valley).

DISTRIBUTION. Type locality: Yakutia: Yakutsk env., Kantagai. Palaearctic: Russia (Yakutia). Conditional endemic, boreal.

53. Dolichopus kjari Stackelberg, 1929

REFERENCES. Stackelberg, 1929: 174 (C Yakutia: Yakutsk);
Grichanov, Bagachanova, 2006: 132 (C Yakutia: Lena-Amga interfluve, Lena River valley).

MATERIAL EXAMINED. C Yakutia: $30^{7}$, Yakutsk, 19, 21.07.1987, Zlobin (ZIN); 10', Khangalassky ulus, Elanka, 130 km SW Yakutsk, 30.06.2005, Bagachanova (IBPC).

DISTRIBUTION. Type locality: Russia: "aus verschiedenen Orten des Distrikts Jakutsk". Palaearctic: Russia (Buryatia, Chita, Irkutsk, Kemerovo, Krasnoyarsk, "Sayan Mountains", Yakutia), Mongolia. East-Siberian boreal.

HABITATS. Forb communities on slope.

\section{Dolichopus latilimbatus Macquart, 1827}

REFERENCES. Negrobov et al., 2013: 58 (Yakutia).

REMARKS. Yakutia was in error included in the area of the species by Negrobov et al. [2013] who erroneously cited Stackelberg [1930a] in their species list. Subsequently, this error was repeated in several more lists.

\section{Dolichopus latipennis Fallén, 1823}

REFERENCES. Stackelberg, 1933: 112 (C Yakutia: Yakutsk); Grichanov, Bagachanova, 2006: 132 (C Yakutia: Lena-Amga interfluve, Lena River valley); Negrobov et al., 2009: 43 (C Yakutia: Lena River valley).

MATERIAL EXAMINED. W Yakutia: $30^{7}$, Suntarsky District, vil. Kutana, 2.07.1987, Zlobin (ZIN); $90^{\top}, 8{ }^{\circ}$, Kyukei Lake, vil. Tumul env., $60 \mathrm{~km} \mathrm{NE}$ vil. Suntar, N 6243'38", E 117²4'44", 27.06.2011, Sivtseva (IBPC); 1+, Mokhsogollookh Lake, env. vil.

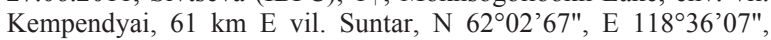
25.06.2011, Sivtseva (IBPC); C Yakutia: 1ㅇ, Oktemtsy, island of Lena River, 27.06.1977, Kaimuk (IBPC); $10^{7}$, Yakutsk, 21.07.1987, Zlobin (ZIN); 1으, Elanka, 30.06.2007; Amga River valley: Mikhailovka, 22.06.1986, Bagachanova (IBPC); $20^{7}$, Aldan River, vil. Megino-Aldan, 5.06.1983, 13.07.1983, Bagachanova (IBPC).

DISTRIBUTION. Type locality: [Sweden: ] "Paroeciae Farhult Scaniae". Palearctic: ?Austria, Belgium, Czech, Denmark, Estonia, Finland, France, Germany, Ireland, Latvia, Netherlands, Norway, Poland, Sweden, UK, N Russia (Karelia, Murmansk, Nenets), E Russia (Buryatia, Irkutsk, Kamchatka, Novosibirsk, Republic of Altai, Sakhalin, Sayan Mnt., Yakutia), Mongolia, China (Qinghai); Nearctic: Canada (Quebec, Northwest Terr.). Holarctic temperate. New for W Yakutia.

HABITATS. Cereals, flowering alfalfa; Carex, Scolochloa, Potentilla hygrophyte communities.

\section{Dolichopus lenensis}

Negrobov, Barkalov et Selivanova, 2014*

MATERIAL EXAMINED. C Yakutia: Lena River valley. $10^{7}$, Vladimirovka, $23 \mathrm{~km} \mathrm{~S}$ Yakutsk, 28.06.07 (ZIN).

DISTRIBUTION. Type locality: Russia: Irkutsk government, river Lena. Palaearctic: Russia (Irkutsk, Yakutia). EastSiberian boreal. New for Yakutia.

HABITATS. Hedysarum communities, eastern slope.

\section{Dolichopus lepidus Staeger, 1842*}

MATERIAL EXAMINED. W Yakutia: 10', Sordonnokh Lake, 3 km NW vil. Kempendyai, N 6202'67", E 118³6'03", 24.06.2011, Sivtseva (IBPC); S Yakutia: 107, Malyi Nimnyr, $97 \mathrm{~km}$ NE Chulman vil., point 2, 10.07.2010, Potapova (IBPC); SW Yakutia: 10" Biryuk River, 5 km from Melichan River mouth, 14.07.2008, Bagachanova (IBPC).

DISTRIBUTION. Type locality: Denmark: Slutningen, ved Leersøen. Palaearctic: Austria, Belarus, Belgium, Bosnia and Herzegovina, China (Shaanxi, Beijing), Czech Republic, Denmark, Estonia, Finland, France, Georgia, Kazakhstan, Latvia, Mongolia, Netherlands, Norway, Poland, Romania, Russia (Adygea, Karelia, Khabarovsk, Khantia-Mansia, Krasnodar, Krasnoyarsk, Leningrad, Magadan, Moscow, Murmansk, Novgorod, Pskov, Ryazan, Sayan Mountains, Vladi- 
vostok, Vologda, Voronezh, Yakutia), Slovakia, Spain, Sweden, Switzerland, Turkey (Kars), UK; Oriental: China. TransEurasian temperate. New for Yakutia.

HABITATS. Riparian communities; Carex at bank of lakes and rivers.

\section{Dolichopus linearis Meigen, 1824}

REFERENCES. Stackelberg, 1930a: 64 (C Yakutia: Yakutsk); Grichanov, Bagachanova, 2006: 129 (C Yakutia: Lena-Amga interfluves; SW Yakutia)

MATERIAL EXAMINED. W Yakutia: 10', Mirnyi, dredge area, factory N5, pile, 8.07.2001, Nogovitsyna (IBPC); C Yakutia: Lena River valley: $10^{7}$, Tabaginskii cape, $38 \mathrm{~km}$ Pokrovsky road, branch, Malaise trap, 11-13.08.2010, Bagachanova (IBPC); $30^{\top} \mathrm{O}^{2}$, same label, 18.08.2010; 10', 19, Elanka, 28.06.2008; 10', $20 \mathrm{~km} \mathrm{~S}$ vil. Bulgunnakhtakh, 12.07.1977, Bagachanova (IBPC); 10 River, vil. Megino-Aldan, 13.07.1983, Bagachanova (IBPC); $10^{\text {T}}$, 1, Amga River, Syrdyk locality, $25 \mathrm{~km}$ from vil. Bolugur, Malaise trap, 16.11-13.07.2009, Bagachanova (IBPC); 10 , Amginsky District, Mikhailovka, 24.07.1987, Zlobin (ZIN); SW Yakutia: $20^{7}, 1$ 우, Biryuk River mouth, 11.07.2008; $20^{7}$, Biryuk River, $4 \mathrm{~km}$ from Melichan River mouth, 13.07.2008; 10 , 39, Lena River left bank, env. vil. Solyanka, 5 km NE Olekminsk, 24.08.2008; $50^{7}$, 49, Biryuk River, water post, $18 \mathrm{~km}$ from mouth, 16.07.2008, Bagachanova (IBPC); 107, Lensky District, vil. Tolon, 14.07.1987, Zlobin (ZIN).

DISTRIBUTION. Type locality: not given. Palaearctic: Austria, Belgium, China (Heilongjiang, Jilin, Beijing, Inner Mongolia, Gansu, Xinjiang, Qinghai), Czech Republic, Denmark, Estonia, Finland, France, Georgia, Germany, Hungary, Ireland, Italy, Kazakhstan, Latvia, Mongolia, Netherlands, Norway, Poland, Romania, Russia (Adygea, Altai Rep., Krasnodar, Leningrad, Novgorod, Pskov, Ryazan, Vologda, Voronezh, Baikal, Irkutsk, Khabarovsk, Khantia-Mansia, Krasnoyarsk, Yakutia, Magadan, Kamchatka, Amur Reg., Vladivostok, Sakhalin), Slovakia, Sweden, Switzerland, UK. TransEurasian temperate. New for W Yakutia.

HABITATS. Riparian communities; forb meadow; willow bed, cereals, Sanguisorba, Ranunculus, Carex, Rumex communities; meso-hygrophyte, hygrophyte, at rocks, pebblestone, swamp, bank of lakes and rivers.

\section{Dolichopus lineatocornis Zetterstedt, 1843*}

MATERIAL EXAMINED. 107, C Yakutia: Lena River valley: Tabaginskii cape, Malaise trap, 23-25.06.2010, Bagachanova (IBPC)

DISTRIBUTION. Type locality: Sweden: Lund. Palaearctic: Belgium, Czech, Denmark, Estonia, Finland, Germany, Kazakhstan, Latvia, Lithuania, Netherlands, Russia (Krasnodar, Lipetsk, Pskov, Tver, Voronezh, Yakutia), Sweden, UK. Euro-Siberian temperate. New for Yakutia.

HABITATS. Agropyron steppe community; at slope.

\section{Dolichopus litorellus Zetterstedt, 1852}

REFERENCES. Stackelberg, 1933: 65 (C Yakutia: Yakutsk); Negrobov, Chalaya, 1991: 72 (C Yakutia: Lena River valley); Grichanov, Bagachanova, 2006: 132 (C Yakutia: Lena-Amga interfluve, Lena River valley).

MATERIAL EXAMINED. NE Yakutia: $10^{7}, 19$, Indigirka River, $4.5 \mathrm{~km}$ downstream from Kuidusun River mouth, N 63⒉ $33^{\prime} 57^{\prime \prime}$, 143ํำ'13" E, 12.07.2011, Bagachanova (IBPC); 19, old Magadan road between rivulets Small Begel Haya and Big Begel Haya, N $63^{\circ} 15^{\prime} 20^{\prime \prime}, 143^{\circ} 43^{\prime} 33^{\prime \prime}, 18.07 .2011$, Bagachanova (IBPC); 19, Indigirka River, $9 \mathrm{~km}$ downstream from Sarba River, N 63⒊'23", E 142³7’02", 16.07.2011, Bagachanova (IBPC); C Yakutia: LenaAmga interfluve: 1+, Tyungyulyu, 1996; Lena River valley: $10^{2}, 4$ 우, Elanka, 30.06.2008, Bagachanova (IBPC); $10^{2}, 2+$, Pokrovskii road, $38 \mathrm{~km}, 16.08 .2007$, Evdokarova (IBPC); 20 2 , 2 , $4 \mathrm{~km} \mathrm{~W}$ Yakutsk, 8.08.2007, Evdokarova (IBPC); 23 km SW Yakutsk, 6.08.2007, Ermakova (IBPC); 407, 4+, Khatassy, $10 \mathrm{~km} \mathrm{SW} \mathrm{Yakutsk,} \mathrm{16.07.2006,}$ Bagachanova (IBPC); $10^{7}, 18 \mathrm{~km}$ SW Yakutsk, N 61 ${ }^{\circ} 52^{\prime}$, E $129^{\circ} 30^{\prime} 79^{\prime \prime}, 8.07 .2006$, Bagachanova (IBPC); 10', vil. Maimaga, 37 $\mathrm{km} \mathrm{N}$ vil. Namtsy, 17.07.2003, Bagachanova (ZIN); $10^{7}$, Amginsky District, Mikhailovka, 7.08.1987, Zlobin (ZIN); SW Yakutia: $50^{7}$, 2 , Lena River left bank, 2nd Neryuktyaiinsk, 35 km SW Olekminsk, 23.07.2008, Bagachanova (IBPC); $10^{7}$, Biryuk River mouth, 11.07.2008, Bagachanova (IBPC); $10^{7}$, vil. Abaga, $23 \mathrm{~km} \mathrm{SW}$ Olekminsk, 21.07.2008, Bagachanova (IBPC)

DISTRIBUTION. Type locality: Sweden: Lomma Scaniae. Palaearctic: Abkhazia, Austria, Azerbaijan, Belarus, Belgium, Bulgaria, Czech Republic, Denmark, Estonia, France, Germany, Hungary, Iran, Ireland, Italy, Kazakhstan, Mongolia, Netherlands, Poland, Romania, Russia (Karachai-Cherkessia, Krasnodar, Kursk, Moscow, Orel, Pskov, Rostov, Ryazan, Ural, Voronezh, Altai, Omsk, Yakutia), Slovakia, Spain, Sweden, Switzerland, Turkey (Kocaeli- zmit), UK, Ukraine (Ternopil), Uzbekistan. Trans-Eurasian temperate. New for NE and SW Yakutia.

HABITATS. Steppe community at slope; cereal-forb meadow; Caltha-Hordeum community on meadow; pasture; Sparganium at lake; legumes, Pinus pumila, Leontopodium alpinum, Veronica, Epimedium, Campanula, Pedicularis, Thymus, Carex, Equisetum; hygrophyte, mesophyte.

\section{Dolichopus longicornis Stannius, 1831}

REFERENCES. Stackelberg, 1933: 66 (C Yakutia: Yakutsk); Negrobov, Chalaya, 1991: 72 (W Yakutia; C Yakutia: Lena River valley); Grichanov, Bagachanova, 2006: 133 (C Yakutia: Lena River valley; SW Yakutia).

MATERIAL EXAMINED. NE Yakutia: $10^{7}$, Cherskii, Kolyma River, 18.08.1971, Chelnokov (ZIN); $20^{7}$, Yakutsk, 21.07.1987, Zlobin (ZIN); Khaiysardakh, $34 \mathrm{~km}$ NE Batagai vil., 3.07.2009, Potapova (IBPC). W Yakutia: $30^{\top}, 1+$, Mirnyi, dredge factory, pile, 8.07.2001, Nogovitsyna (IBPC); C Yakutia: $50^{7}$, Amginsky District, Mikhailovka, 30.07, 31.07, 7.08, 8.08.1987, Zlobin (ZIN); Lena River valley: 19 , Elanka, 28.06.2008; Middle Lena River, 20 $\mathrm{km} \mathrm{S}$ vil. Bulgunnakhtakh, 12.07.1977, Bagachanova (IBPC); 1웅 Amga River, env. Syrdyk vil., $25 \mathrm{~km}$ from vil. Bolugur, 16.07.2009, Bagachanova (IBPC); 1으. Labyda Lake, $17 \mathrm{~km}$ NW vil. Sitte, 24.07.2012, Sivtseva (IBPC); SW Yakutia: 1 $0^{7}$, env. vil. Solyanka, 5 km N Olekminsk, 25.07.2008, Bagachanova (IBPC); $10^{7}$, Lensky District, vil. Tolon, 14.07.1987, Zlobin (ZIN).

DISTRIBUTION. Type locality: not given [Germany]. Palaearctic: Austria, Belarus, Belgium, China, Czech, Denmark, Estonia, Finland, France, Germany, Hungary, Ireland, Italy, Kazakhstan, Latvia, Lithuania, Luxembourg, Mongolia, Netherlands, Norway, Poland, Romania, Russia (Altai Rep., Amur Reg., Arkhangelsk, Irkutsk, Kamchatka, Karelia, Komi, Krasnodar, Krasnoyarsk, Kuril Is., Leningrad, Lipetsk, Magadan, Moscow, Murmansk, Novgorod, Perm, Primorskii Terr., Pskov, Sakhalin, Sayany, Ural, Vologda, Voronezh, Yakutia, Yaroslavl), Serbia, Slovakia, Sweden, Switzerland, UK, Ukraine (Kherson, Carpathians); Nearctic: Canada (Yukon), USA (Alaska). Holarctic temperate. New for NE Yakutia.

HABITATS. Deciduous light forest; at rocks; cereal-forb community at slope; forb meadow; Ranunculus at water, Carex.

\section{Dolichopus maculicornis Verrall, 1875}

Dolichopus consobrinus Zetterstedt, 1859.

REFERENCES. Negrobov et al., 2000a: 84 (as D. consobrinus Ztt.) (Yakutia)

DISTRIBUTION. Type locality: Torringe-lund, Skabersjo Scaniae, Lund, Fogelsang ad Ilstorp, Scan. [Sweden; automatic]. Palaearctic: Germany, Mongolia, Romania, Russia (Buryatia, Chita, Irkutsk, ?Yakutia), Slovakia, Sweden. Euro-Siberian temperate.

REMARKS. We didn't find publications with the original material for this species collected from Yakutia. It is 
possible that Yakutia was in error included in the species area (Negrobov et al., 2000a), which was repeated in a number of the subsequent publications.

62. Dolichopus maculipennis Zetterstedt, 1843

REFERENCES. Grichanov, Bagachanova, 2006: 133 (C Yakutia: Lena River valley).

MATERIAL EXAMINED. N Yakutia: 10 Pokhodsk, $40 \mathrm{~km}$ NW Cherskii, 6.07.1980, Bagachanova (IBPC).

DISTRIBUTION. Type locality: Scandinaviae septentrionalis, Jamtlandia boreali ad Skalstugan; Nordlandia Norvegicae, ad Bjorkvik, Hacksten, Schiervo, Giebostad [Sweden, Norway]. Palaearctic: Denmark, Estonia, Finland, Germany, Norway, Russia (Altai Rep., Kamchatka, Leningrad, Magadan, Murmansk, Yakutia), Sweden, UK; Nearctic: USA: Alaska. Holarctic, Arctic-boreal. New for N Yakutia.

HABITATS. Hygrophyte community.

\section{Dolichopus mannerheimi Zetterstedt, 1838*}

MATERIAL EXAMINED. C Yakutia: 10', D'am, 4 km N vil. Amga, 3.06.1985, Maksimova (IBPC).

DISTRIBUTION. Type locality: Sweden: "Lapponia Umensi, Stensele; Tresunda; Naestansjo; in paroecia Wilhelmina" [= Vilhehnina]. Palaearctic: China (Xinjiang, Heilongjiang), Finland, Mongolia, Norway, Russia (Altai Rep., Buryatia, Chita, Irkutsk, Kamchatka, Karelia, Khantia-Mansia, Khabarovsk, Krasnoyarsk, Magadan, Murmansk, Vladivostok, Yakutia), Sweden; Nearctic: Canada: Yukon; USA: Alaska. Holarctic temperate. New for Yakutia.

64. Dolichopus nataliae Stackelberg, 1930

REFERENCES. Negrobov, Chalaya, 1991: 73 (NE Yakutia). DISTRIBUTION. Type locality: Russia: "Sibirie orientalis, prov. Austro-Ussuriensis, distr. Sutchaniensis; via Spassk-Jakovlevka, fl. Ugodzina, distr. Spasskensis, Tigrovaja”. Palaearctic: Russia (Khabarovsk, Magadan, Vladivostok, Yakutia). North-East-Siberian-Far-Eastern temperate.

65. Dolichopus negrobovi Gosseries, 1989

REFERENCES. Grichanov, 2002: 123 (C Yakutia: Lena-Amga interfluve); Grichanov, Bagachanova, 2006: 133 (NE Yakutia; C Yakutia: Lena-Amga interfluve, Lena River valley).

MATERIAL EXAMINED. C Yakutia: $10^{7}$, Khangalassky ulus, Malaya Keteme River, 145 km SW Yakutsk, 30.06.2005, Bagachanova (IBPC).

DISTRIBUTION. Type locality: Russia: "Burjatskaja ASSR, Ustj-Bargusinskaja Tal, Jareehta”. Palaearctic: Mongolia, Russia (Blagoveshchensk, Buryatia, Irkutsk, Kamchatka, Khabarovsk, Magadan, Vladivostok, Yakutia). East-Siberian-Far-Eastern temperate.

HABITATS. Forb community.

\section{Dolichopus notatus Staeger, 1842}

REFERENCES. Negrobov, Chalaya, 1991: 73 (C Yakutia: Lena River valley).

DISTRIBUTION. Type locality: Denmark: "I Moser; Dyrehaven og: Engene ved Leersøen, temmelig fjelden". Palaearctic: Austria, Belgium, Czech, Denmark, Estonia, Finland, France, Germany, Hungary, Iran, Kazakhstan, Netherlands, Norway, Poland, Romania, Russia (Altai Rep., Blagoveshchensk, Karelia, Khabarovsk, Leningrad, Magadan, Moscow, Ryazan, Vladivostok, Voronezh,Yakutia), Slovakia, Sweden, Switzerland, UK. Trans-Palaearctic temperate.

67. Dolichopus aff. orientalis Parent, 1927 MATERIAL EXAMINED. NE Yakutia: $1 \sigma^{\gamma}$, Cherskii Ridge,
Moma River head, 50 km E Pobeda Mnt., env. vil. Sasyr, 23.07.1998, Nogovitsyna (ZIN).

REMARKS. The male examined belongs most probably to an undescribed species.

\section{Dolichopus pennatus Meigen, 1824}

REFERENCES. Negrobov, Chalaya, 1991: 73 (C Yakutia: Lena River valley).

MATERIAL EXAMINED. SW Yakutia: 10', Biryuk River, 5 $\mathrm{km}$ from Melichan River mouth, 14.07.2008, Bagachanova (IBPC).

DISTRIBUTION. Type locality: not given. Palaearctic: Austria, Belarus, Belgium, Bosnia and Herzegovina, Bulgaria, Czech, Denmark, Estonia, Finland, France, Georgia, Germany, Hungary, Ireland, Italy, Latvia, Lithuania, Mongolia, Netherlands, Norway, Poland, Portugal, Romania, Russia (Adygea, Alania, Altai Rep., Altai Terr., Arkhangelsk, Blagoveshchensk, Chechnya, Irkutsk, Kabardino-Balkaria, Karachai-Cherkessia, Karelia, Khabarovsk, Krasnodar, Krasnoyarsk, Kuril Is., Leningrad, Magadan, Moscow, Murmansk, Novgorod, Pskov, Sakhalin, Tatarstan, Vladivostok, Vologda, Voronezh, Yakutia), Slovakia, Spain, Sweden, Switzerland, Turkey (Kars), UK, Ukraine. Trans-Eurasian temperate. New for SW Yakutia.

HABITATS. Riparian community, Carex.

\section{Dolichopus planitarsis Fallén, 1823}

REFERENCES. Grichanov, Bagachanova, 2006: 134 (C Yakutia: Lena River valley).

DISTRIBUTION. Type locality: Sweden: "Borringe Scaniae". Palaearctic: Austria, Belarus, Belgium, Czech, Denmark, Estonia, Finland, France, Georgia, Germany, Hungary, Ireland, Mongolia, Netherlands, Norway, Poland, Russia (Altai Rep., Kamchatka, Krasnoyarsk, Leningrad, Voronezh, Yakutia), Slovakia, Sweden, Switzerland, UK. TransEurasian temperate.

\section{Dolichopus platychaetus \\ Negrobov et Barkalov, 1977*}

MATERIAL EXAMINED. NE Yakutia: $1 \sigma^{\prime}, 300 \mathrm{~km}$ ENE vil. Khandyga, Suntar River lower reaches, at water post, Indigirka River basin, 19.07.1985, L.I. Popova (ZIN).

DISTRIBUTION. Type locality: Russia: Magadan region, river Khinikandzha. Palaearctic: Mongolia, Russia (Magadan, Yakutia). East-Siberian temperate. New for Yakutia.

71. Dolichopus plumipes (Scopoli, 1763)

REFERENCES. Negrobov, Chalaya, 1991: 73 (C Yakutia: Lena River valley); Grichanov, Bagachanova, 2006: 134 (NW and W Yakutia; C Yakutia: Lena-Amga interfluve, Lena River valley).

MATERIAL EXAMINED. NE Yakutia: Oimyakonskoe upland, Labynkyr Lake, E bank, 1.07.2012, Popov (IBPC); W Yakutia: 1웅, Nyurba, 19.06.1986, Kaimuk (IBPC); 19 , Mirnyi, dredge area, factory N5, pile, 8.07.2001, Nogovitsyna (IBPC); 10, 3ㅇ, Kyukei Lake, vil. Tumul env., $60 \mathrm{~km}$ NE vil. Suntar, N 6243'38", E 117²5'44", 27.06.2011, Sivtseva (IBPC); 2, Mokhsogollookh Lake, env. vil. Kempendyai, 61 km E vil. Suntar, N 6201'62", E 118³9'07", 25.06.2011, Sivtseva (IBPC); Lena-Amga interfluve: $10^{7}$, Borogontsy, 96 km NE Yakutsk, alas, 11.07.2008, Bagachanova (IBPC); Lena River valley, 1 을 Oktemtsy, 17.06.1977; $2 \sigma^{\top}$, Elanka, 30.06.2008; $2+$, same label, 28.06.2008; 1 을 Tabaginskii cape, Malaise trap, 28 29.07.2009, Bagachanova (IBPC); 19, Botanical Garden, 10.08.2005, Evdokarova (IBPC); Amga River valley: 1 ㅇ, Mikhailovka, 22.06.1986, Bagachanova (IBPC). SW Yakutia: $4{ }^{7}, 4$, Lena River left tributary, Biryuk River mouth, 11.07.2008, Bagachanova (IBPC); 2 , Biryuk River, $4 \mathrm{~km}$ from Milichan River mouth, 13.07.2008; 1의, Biryuk River mouth, 11.07.2008; 10', Biryuk River mouth, 11.07.2008; $1 \sigma^{7}, 6+$, Biryuk River, Lena River left tributary, $4 \mathrm{~km}$ from mouth Milichan River, 13, 14.07.2008; 50', 9ㅇ, env. vil. Solyanka, 5 km N Olekminsk, 24.07.2008; 10', 1의, vil. Abaga, 23 km 
SW Olekminsk, Khomustakh Lake, 20.07.2008, Bagachanova (IBPC); S Yakutia: $3 \sigma^{7}, 2$, Malyi Nimnyr, $97 \mathrm{~km}$ W vil. Chulman, 10.07.2010, Nogovitsyna (IBPC).

DISTRIBUTION. Type locality: Slovenia: "Carnioliae indigena". Asia (Afghanistan, Palaearctic China, Kazakhstan, Mongolia, Turkey, Uzbekistan, Russia: Baikal, Chukotka, Irkutsk, Kamchatka, Khabarovsk, Khantia-Mansia, Koryakia, Novosibirsk, Tomsk, Tyumen, Vladivostok), Caucasus, Europe; Nearctic, Neotropical and Oriental Regions. Multiregional polyzonal. New for S and SW Yakutia.

HABITATS. Riparian communities, sedge-grass community, swamp meadow, forb meadow, steppe slope, at fir grove, pebblestone, willow bed, swamp with birches, flowering alfalfa, hummocky tundra; cereals, Rumex, Carex, Scolochloa, Ranunculus, Sanguisorba; mesophyte, mesohygrophyte, hygrophyte communities.

72. Dolichopus plumitarsis Fallén, 1823

REFERENCES. Stackelberg, 1933: 83 (C Yakutia: Yakutsk); Negrobov, Chalaya, 1991: 74 (C Yakutia: Amga River valley).

DISTRIBUTION. Type locality: Sweden. Palaearctic: Austria, Belarus, Belgium, Bulgaria, China (Heilongjiang, Hebei, Beijing, Inner Mongolia, Xinjiang), Czech, Estonia, Finland, Germany, Italy, N Kazakhstan, Netherlands, Norway, Poland, Russia (Altai Rep., Buryatia, Chita, Commander Is., Karachai-Cherkessia, Irkutsk, Khabarovsk, Krasnodar, Krasnoyarsk, Kuriles, Moscow, Perm, Sakhalin, Tomsk, Ural, Vladivostok, Voronezh, Yakutia), Slovakia, Sweden, Switzerland, UK; Nearctic: Canada (Ontario), USA (Alaska). Holarctic temperate.

\section{Dolichopus pospelovi Smirnov, 1948}

REFERENCES. Grichanov, Bagachanova, 2006: 134 (NW Yakutia)

MATERIAL EXAMINED. C Yakutia: $2 \sigma^{\top}, 2 \circ$, Tabaginskii cape, Malaise trap, 28-30.07.2009; NW Yakutia: 10', Muna River valley (Lena River left tributary), RR «Muna», Malaise trap, 1113.08.2005, Stepanov (ZIN)

DISTRIBUTION. Type locality: Russia: Buchta Ta-Chingauz, river, Sudzukhinski reserve [= Lazovski reserve]. Palaearctic: Russia (Vladivostok, Yakutia). East-Siberian-FarEastern temperate. New for C Yakutia.

HABITATS. Under slope at branch.

\section{Dolichopus punctum Meigen, 1824}

REFERENCES. Grichanov, Bagachanova, 2006: 134 (C Yakutia: Lena River valley; S Yakutia).

DISTRIBUTION. Type locality: Germany: "Gegend von Berlin". Palaearctic: Austria, Finland, Germany, Poland, Russia (Khabarovsk, Leningrad, Moscow, Yakutia), Sweden. Trans-Eurasian temperate.

\section{Dolichopus ringdahli Stackelberg, 1930}

REFERENCES. Stackelberg, 1930b: 160 (C Yakutia); Stackelberg, 1933: 88 (C Yakutia); Negrobov, Chalaya, 1991: 74 (C Yakutia: Lena-Amga interfluve).

DISTRIBUTION. Type locality: Russia: "Kreis Jakutsk: Keedej-See; Sud-Ussuri-Gebiet: Tigrovaya. Kreis Sutshan". Palaearctic: Russia (Buryatia, Vladivostok, Yakutia), China (Jilin). East-Siberian-Far-Eastern temperate.

\section{Dolichopus rotundipennis Loew, 1848}

REFERENCES. Negrobov, Chalaya, 1991: 74 (C Yakutia: Lena River valley); Grichanov, 2002: 123 (C Yakutia: Lena-Amga interfluve); Grichanov, Bagachanova, 2006: 134 (C Yakutia: LenaAmga interfluve).

DISTRIBUTION. Type locality: [Russia:] "Sibirien". Palaearctic: China (Qinghai), Kazakhstan, Mongolia, Russia
(W Siberia, Sayan Mtns., Buryatia, Chita, Yakutia), Tajikistan. Siberian-Mongolian temperate.

\section{Dolichopus rupestris Haliday, 1833}

REFERENCES. Stackelberg, 1933: 90 (C Yakutia: Yakutsk); Negrobov, Chalaya, 1991: 74 (C Yakutia: Lena-Amga interfluve; Lena River valley); Grichanov, Bagachanova, 2006: 134 (NE and S Yakutia).

MATERIAL EXAMINED. NE Yakutia: $1 \sigma^{7}$, Cherskii, River bank, 6.08.1972, Gorodkov (ZIN); $10^{7}$, Verkhoyanskii Ridge, Ilderkei Lake, Orto-Sala river head, $970 \mathrm{~m}$ a. s. 1., 18.07.1991, Vinokurov (IBPC); $1 \sigma^{7}$, Verkhoyanskii Ridge, Kele River head, Nimnyr, Kokchin River head, 3.07.1991, Bagachanova (IBPC); 29 , 15 km NE vil. Khaiysardakh, 18.07.2010, Popova (IBPC); SW Yakutia: $1 \sigma^{7}$, Biryuk River, $18 \mathrm{~km}$ from mouth, water post, 16.07.2008, Bagachanova (IBPC); S Yakutia: 20', Aldan upland, Kurun-Khoonku River mouth, left tributary of Timpton River, 2 km from mouth, 15, 17.08.2010, Nogovitsyna (IBPC); 19, Aldan upland, Chulman River mouth, right tributary of Timpton River, 5.08.2010, Bagachanova (IBPC); $3 \sigma^{7}$, Malyi Nimnyr, $97 \mathrm{~km}$ NE vil. Chulman, 2nd point, 10.07.2010, Potapova (IBPC).

DISTRIBUTION. Type locality: England: Downshire, Tullymore Park and Mountains of Mourne. Palaearctic: Austria, Belgium, China (Xinjiang), Czech, Denmark, Finland, France, Germany, Ireland, Italy, Kazakhstan, Latvia, Lithuania, Netherlands, Norway, Poland, Russia (Altai Rep., Arkhangelsk, Bering Is., Buryatia, Kamchatka, Karelia, Khabarovsk, Kuril Is., Leningrad, Murmansk, Nenetsia, Voronezh, Vladivostok, Yakutia, Ural), Sweden, Switzerland, UK; Nearctic: Canada, USA. Holarctic temperate. New for SW Yakutia.

HABITATS. Birch-sedge hummocky tundra, sedge-grass meadow.

\section{Dolichopus sagittarius Loew, 1848}

REFERENCES. Negrobov, Chalaya, 1991: 74 (NE Yakutia). Grichanov, 2002: 123 (C Yakutia: Lena-Amga interfluve); Grichanov, Bagachanova, 2006: 134 (C Yakutia: Lena-Amga interfluve).

DISTRIBUTION. Type locality: Russia: Siberia. Palaearctic: China, Mongolia, Russia (Altai Rep., Buryatia, Irkutsk, Magadan, Tomsk, Yakutia); Nearctic: Canada, USA. Siberian-Far-Eastern-Nearctic temperate.

\section{Dolichopus selivanovae \\ Negrobov et Barkalov, 2010*}

MATERIAL EXAMINED. NE Yakutia: 10, Khaiysardakh env., 34 km NE Batagai vil., 3.07.2009, Potapova (ZIN).

DISTRIBUTION. Type locality: Russia: Republic of Altai, Kosh-Agach district, Plateau Ukok, Lake Muzdy-Bulak. Palaearctic: Russia (Altai Rep., Yakutia). Mountains of Altai and Yakutia. New for Yakutia.

\section{Dolichopus sibiricus Stackelberg, 1929}

REFERENCES. Stackelberg, 1929: 169 (C Yakutia: LenaAmga interfluve); Negrobov, Chalaya, 1991: 75 (C Yakutia: Amga River valley; Lena River valley).

DISTRIBUTION. Type locality: Russia: "Kreis Jakutsk; Tongutte-Tordo, bei Amga, linker Nebenflus des Aldan, Keedej-See, 25 km Ostlich von dem rechten Ufer der Lena 2. Zhemkonskij Nasleg, am rechten Ufer der Lena, ungefahr 80 km aufwarts von Jakutsk". Palaearctic: Russia (Yakutia). Conditional endemic, boreal.

\section{Dolichopus simius Parent, 1927}

REFERENCES. Grichanov, Bagachanova, 2006: 135 (SW Yakutia).

MATERIAL EXAMINED. SW Yakutia. 207, Lensky District, vil. Tolon, 14-15.07.1987, Zlobin (ZIN).

DISTRIBUTION. Type locality: Russia: Siberie: environs 
d'Irkutsk. Palaearctic: Russia (Altai Rep., Bashkortostan, Blagoveshchensk, Buryatia, Chita, Commander Is., Irkutsk, Kamchatka, Khabarovsk, Khakassia, Krasnoyarsk, Magadan, Moscow, Novosibirsk, Sakhalin, Sverdlovsk, Tomsk, Vladivostok, Yakutia), Mongolia, China (Heilongjiang, Inner Mongolia). East-European-Siberian-Far-Eastern temperate.

\section{Dolichopus simplex Meigen, 1824}

REFERENCES. Grichanov, 2014: 211 (Yakutia).

REMARKS. Yakutia was probably in error included in the area of the species by Grichanov [2014] in his species list. Subsequently, this error was repeated in several more lists.

\section{Dolichopus socer Loew, 1871}

REFERENCES. Stackelberg, 1933: 97 (C Yakutia: Yakutsk); Negrobov, Chalaya, 1991: 75 (C Yakutia: Amga River valley, Lena-Amga interfluve, Lena-Vilyui interfluve; C Yakutia); Grichanov, Bagachanova, 2006: 135 (C Yakutia: Lena River valley; N, NE, $S$ and $W$ Yakutia).

MATERIAL EXAMINED. NE Yakutia: $20^{7}$, Cherskii, River bank, 6.08.1972, Gorodkov (ZIN); $30^{7}, 3+$, Verkhoyanskii Ridge, Ilderkei Lake, Orto-Sala river head, $970 \mathrm{~m}$ a. s. 1., 18.07.1991, Vinokurov (IBPC); 1을 Kolyma River, Ruzhnikovo, 106 km NE Sroednekolymsk, 1.08.2008, A. Popov (IBPC); 19, Oimyakoesk, Agayakan River and Suntar River, at bridge, 780 m, 2.07.1994 Vinokurov (IBPC); 107, 19, El'ginskoe upland, El'ga River left bank, Indigirka River left tributary, floodplain, Malaise trap, 25.07.2008, Nogovitsyna (IBPC); 10', Oimyakonskoe upland, Labynkyr Lake, S bank, 4.07.2012, Popov (IBPC); $10^{7}, 15 \mathrm{~km}$ NE vil. Khaiysardakh, 17.07.2010, Popova (IBPC). NW Yakutia: $60^{7}, 3$, Ebelyakh, Anabar, 23.07.1988, Gorodkov (ZIN); $70^{7}$, Saskylakh airport, 24.07.1988, Gorodkov (ZIN); 20 , 1\%, Saskylakh, 28.07.1988, Gorodkov (ZIN).

DISTRIBUTION. Type locality: Russia: "Ochotsk". Palaearctic: Kazakhstan, Mongolia, Russia (Altai Rep., Blagoveshchensk, Buryatia, Kamchatka, Khabarovsk, Krasnoyarsk, Magadan, Tomsk, Yamalia, Yakutia). Siberian-FarEastern temperate.

HABITATS. Forest-tundra, cereal-moss meadow, light moss forest, swampy pond, swampy bank of former riverbed, bank of lake; cereal grasses, Chosenia, Populus, Poa, Carex.

\section{Dolichopus taigensis Smirnov, 1948}

REFERENCES. Negrobov, Chalaya, 1991: 75 (C Yakutia: Lena-Amga interfluve; Aldan River basin).

DISTRIBUTION. Type locality: Kluch Podnebesnyi, Sikhote-Alin, Primorye. Palaearctic: Russia (Kamchatka, Karelia, Khabarovsk, Magadan, Vladivostok, Yakutia), ?Uzbekistan. East-European-Siberian-Far-Eastern temperate.

\section{Dolichopus terminasianae}

Negrobov, Selivanova et Maslova, 2011*

MATERIAL EXAMINED. NE Yakutia: $20^{\top}, 19,15 \mathrm{~km}$ NE vil. Khaiysardakh, 17-18.07.2010, Potapova (IBPC); 307, 15 km NE Khaiysardakh, 17-18.07.2010, L.I. Popova (ZIN).

DISTRIBUTION. Type locality: Magadan obl., Aborigen peak, $100 \mathrm{~km} \mathrm{~N}$ Ust'-Omchug, Annachag ridge, Olen' river. Palaearctic: Russia (Magadan, Yakutia). North-EastSiberian boreal. New for Yakutia.

HABITATS. Hummocky tundra, birch-sedge communities, cereal-moss meadow, light moss forest.

\section{Dolichopus ungulatus (Linnaeus, 1758)*}

MATERIAL EXAMINED. SW Yakutia: 1옹 Peledui Rive mouth, Lena River left tributary, 16.07.2010, Burnashova (IBPC)

DISTRIBUTION. Type locality: Europe. Palaearctic: Austria, Belarus, Belgium, Bulgaria, China (Xinjiang), Croatia, Czech Republic, Denmark, Estonia, Finland, France, Georgia, Germany, Hungary, Iran, Ireland, Italy, Kazakhstan, Latvia,
Lithuania, Luxembourg, Netherlands, Norway, Poland, Romania; Russia (Adygea, Alania, Altai Rep., Altai Terr., Chechnya, Irkutsk, Kabardino-Balkaria, Karachai-Cherkessia, Karelia, Khabarovsk, Khantia-Mansia, Krasnodar, Krasnoyarsk, Kursk, Leningrad, Lipetsk, Mordovia, Moscow, Novgorod, Novosibirsk, Orenburg, Perm, Pskov, Ryazan, Tatarstan, Vologda, Voronezh, Yakutia), Serbia, Slovakia, Spain, Sweden, Switzerland, UK, Ukraine (Kharkiv, Kyiv, Odessa, Ternopil, Carpathia). Trans-Eurasian. New for Yakutia.

\section{Dolichopus uniseta Stackelberg, 1929}

REFERENCES. Stackelberg, 1929: 177 (C Yakutia: Yakutsk); Stackelberg, 1933: 103 (C Yakutia: Yakutsk); Negrobov, Chalaya, 1991: 75 (C Yakutia: Amga River valley, Lena-Amga interfluve); Grichanov, Bagachanova, 2006: 135 (C Yakutia: Lena River valley).

MATERIAL EXAMINED. C Yakutia: $10^{7}$, Kuranakh Rivermouth, $16 \mathrm{~km}$ upstream from Labyia River, 4.07.2001, Potapova (ZIN).

DISTRIBUTION. Type locality: Russia: "Kreis Jakutsk: Olom; Abyj, ungefahr $60^{\circ} 50^{\prime}$ nordlicher Breite und $130^{\circ}$ Ostlicher Lange zwischen der Lena und Amga, Amginiskaja Sloboda, amlinken Ufer des Flukes Amga; Sud-Ussuri-Gebiet: Jakovlevka, Kreis Spassk". Palaearctic: Russia (Khabarovsk, Vladivostok, Yakutia), China (Heilongjiang, Hebei, Beijing, Shaanxi); Oriental: China. East-Siberian-Far-Eastern temperate.

HABITATS. Sedge meadow.

\section{Dolichopus vitripennis Meigen, 1824}

REFERENCES. Grichanov, 2012: 317 (NW and C Yakutia). MATERIAL EXAMINED. NW Yakutia: $10^{7}$, Ebelyakh, Anabar River bank, 22.07.1988, Gorodkov (ZIN); NE Yakutia: 107, 1 $\mathrm{km}$ NE vil. El'gets, tourist base, 17.07.2010, Popova (IBPC); $10^{7}$, Yana-Adycha interfluve between vil. Khaiysardakh and Kisilyakh, 28.07.2009, Potapova (IBPC); C Yakutia: $1 \sigma^{\prime}$, Yakutsk, 18.07.1969, Sychevskaya (ZIN).

DISTRIBUTION. Type locality: not given. Palaearctic: Austria, Belgium, Czech, Denmark, Estonia, Finland, France, Germany, Hungary, Ireland, Italy, Latvia, Netherlands, Norway, Poland, Portugal, Romania, Slovakia, Spain, Sweden, Switzerland, UK, Russia (Karelia, Leningrad, Moscow, Orenburg, Yakutia), North Kazakhstan. Euro-Siberian temperate. New for NE Yakutia.

HABITATS. Moss-shrub forest, willow bed, cereal-sedge meadow.

\section{Dolichopus zetterstedti Stenhammar, 1852}

REFERENCES. Stackelberg, 1933: 108 (C Yakutia: Yakutsk); Negrobov, Chalaya, 1991: 76 (C Yakutia: Amga River valley).

DISTRIBUTION. Type locality: Sweden: Ringstad. Palaearctic: Finland, N Kazakhstan, Norway, Russia (West Siberia, Yamalia, Yakutia), Sweden. Euro-Siberian boreal.

\section{Dolichopus zhelochovzevi Negrobov, 1976}

REFERENCES. Negrobov, 1976b: 512 (C Yakutia: Yakutsk); Grichanov, Bagachanova, 2006: 130 (C Yakutia: Lena-Amga interfluve, Lena River valley).

MATERIAL EXAMINED. C Yakutia: 10', Yakutsk, 5.07.2000, Bagachanova (ZIN); W Yakutia: $20^{7}$, Suntarsky District, vil. Kutana, 2.07.1987, Zlobin (ZIN).

DISTRIBUTION. Type locality: Russia: near Yakutsk. Palaearctic: Russia (Yakutia). Conditional endemic, boreal. New for W Yakutia.

\section{Hercostomus flavicoxus} Negrobov et Logvinovskij, 1977

REFERENCES. Negrobov, Chalaya, 1991: 75 (C Yakutia: Lena River valley).

MATERIAL EXAMINED. C Yakutia: 20 $0^{7}$, Amginsky District, Mikhailovka, 24, 25.07.1987, Zlobin (ZIN). 
DISTRIBUTION. Type locality: Russia: "2 Zhermunskij nochleg, Yakut ASSR". Palaearctic: Russia (Yakutia). Conditional endemic, boreal.

\section{Hercostomus gracilis (Stannius, 1831)}

REFERENCES. Grichanov, 2014: 189 (Yakutia).

REMARKS. Yakutia and other territories of Siberia and Russian Far East were in error included in the area of the species by Grichanov [2014] in his species list. Subsequently, this error was repeated in several more lists.

\section{Hercostomus rusticus (Meigen, 1824)}

REFERENCES. Grichanov, Bagachanova, 2006: 135 (C Yakutia. Amga River valley).

MATERIAL EXAMINED. C Yakutia: Lena River valley, $10^{7}$ 1ㅇ, Oktemtsy, 7, 8.07.1977, Kaimuk (IBPC); $20^{7}$, Amginsky District, Mikhailovka, 2.08.1987, Zlobin (ZIN).

DISTRIBUTION. Type locality: not given. Palaearctic: Abkhazia, Armenia, Austria, Belarus, Belgium, Bosnia and Herzegovina, Bulgaria, Czech, Estonia, France, Georgia, Germany, Greece, Hungary, Iran, Italy, N Kazakhstan, Macedonia, Mongolia, Netherlands, Poland, Romania, Russia (Adygea, Altai Rep., Blagoveshchensk, Buryatia, Chechnya, Crimea, Dagestan, Irkutsk, Kabardino-Balkaria, KarachaiCherkessia, Krasnodar, Krasnoyarsk, Kursk, Leningrad, Novosibirsk, Omsk, Samara, Ural, Vladivostok, Voronezh, Yakutia), Slovakia, Spain, Switzerland, Ukraine (Poltava). TransEurasian temperate.

\section{Hercostomus sviridovae}

Negrobov et Chalaya, 1987*

MATERIAL EXAMINED. C Yakutia: Amga River valley, $20^{7}$, Amginsky District, Mikhailovka, 25.07.1987, Zlobin (ZIN); Lena River valley. $10^{7}$, Yakutsk, Botanical Garden, 20.07.1987, Zlobin (ZIN).

DISTRIBUTION. Type locality: Russia: Primorie, Bikin river. Palaearctic: Russia (Chita, Ulan-Ude, Vladivostok, Yakutia). East-Siberian-Far-Eastern. New for Yakutia.

\section{Hercostomus vivax (Loew, 1857)}

REFERENCES. Negrobov, Chalaya, 1991: 76 (C Yakutia: Lena-Amga interfluve).

DISTRIBUTION. Type locality: Germany: "Nordliche Deutschland". Palaearctic: Austria, Belarus, Belgium, Bulgaria, Czech, Croatia, Denmark, Estonia, France, Georgia, Germany, Hungary, Italy, Latvia, Poland, Romania, Russia (Altai Rep., Karachai-Cherkessia, Krasnodar, Kursk, Leningrad, Moscow, Ural, Yakutia), Slovakia, Spain, Switzerland, Ukraine (Uzhhorod). Euro-Siberian temperate.

\section{Hydrophorus alpinus Wahlberg, 1844}

REFERENCES. Frey, 1915: 63 (N Yakutia); Grichanov, Bagachanova, 2006: 135 (N Yakutia).

MATERIAL EXAMINED. NE Yakutia: $80^{7}$, Saskylakh airport, 24.07.1988, Gorodkov (ZIN); $20^{7}+$, Ebelyakh, Anabar 23.07.1988, Gorodkov (ZIN); $100^{7}$, , Cherskii, Kolyma, River bank, 18.08.1972, Gorodkov, Chelnokov (ZIN); $150^{7}+$, Chai-Tumuss, $125^{\circ} 40,26.07 .1957$, Gorodkov (ZIN); $20^{\circ}+$, Tiksi, $7 \mathrm{~km} \mathrm{~N} \mathrm{r}$ Kharogor-Yurege, 13.08.1957, Gorodkov (ZIN); 1ㅇ, Kyusyur, River lower reaches, $12 \mathrm{~km} \mathrm{NE}$, 21.07.1957, Gorodkov (ZIN).

DISTRIBUTION. Type locality: Sweden: "Walli et Snjerack prope Quickjock". Palaearctic: Finland, Norway, Russia (Arkhangelsk, Chukotka, Leningrad, Murmansk, Nenetsia, Yakutia, Yamalia), Sweden; Nearctic: Canada, USA. Holarctic Arctic-boreal. New for NE Yakutia.

HABITATS. Forest-tundra, swamp meadow, sedge swamp, swamp hollows, bank of rivulets, lakes and ponds, willow bed.

\section{Hydrophorus altivagus Aldrich, 1911*}

MATERIAL EXAMINED. C Yakutia: 107, Tabaginskii cape, 38 km Pokrovsky road, 11-13.08.2010, Bagachanova (ZIN); 10', Tabaginskii cape, $38 \mathrm{~km}$ Pokrovsky road, 13.08.2010, Bagachanova (IBPC); 10, Env. Yakutsk, Botanical garden, 20.05.2012, Burnashova (IBPC); 10', Lena-Vilyui interfluve, Ulakhan Chabyda Lake, $30 \mathrm{~km} \mathrm{~N}$ vil. Magarassy, 6.09.2012, Popov (IBPC); SW Yakutia: 20, Biryuk River, $4 \mathrm{~km}$ from Melichan River mouth, 13.07.2008, Bagachanova (IBPC); 10 $0^{7}$, + , vil. Solyanka, env. Olekminsk, 25.07.2008, Bagachanova (IBPC); NE Yakutia: $70^{7}+$, Cherskii, River bank, 18.08.1972, Gorodkov (ZIN).

DISTRIBUTION. Type locality: USA: Colorado, Marshall Pass. Palaearctic: Finland, Norway, Russia (Kamchatka, Karelia, Leningrad, Mordovia, Murmansk, Yakutia), Sweden; Nearctic: Canada, USA. Holarctic temperate. New for Yakutia.

HABITATS. Willow bed, bank of rivulets, branches and puddles, on snow, forb-cereal communities, sedges.

96. Hydrophorus arcticus Negrobov, 1977

REFERENCES. Grichanov, Bagachanova, 2006: 136 (N Yakutia). DISTRIBUTION. Type locality: Russia: "Nat.-Kreis Tschuktschen, Kaljutschinskaja guba". Palaearctic: Russia (Chukotka, Komi, Yamalia, Yakutia). Euro-Siberian Arctic.

\section{Hydrophorus balticus (Meigen, 1824)}

REFERENCES. Negrobov et al., 2000a: 85 (Yakutia; no material provided).

DISTRIBUTION. Type locality: Germany: Hamburg. Palaearctic: Afghanistan, Algeria, Austria, Azerbaijan, Belgium, Bosnia and Herzegovina, Bulgaria, Cyprus, Czech Republic, Denmark, Estonia, Finland, France, Georgia, Germany, Greece, Hungary, Iran, Ireland, Israel, Italy, Mongolia, Morocco, Netherlands, Norway, Poland, Romania, Russia (Adygea, Alania, Baikal, Chechnya, Kabardino-Balkaria, Karachai-Cherkessia, Krasnodar, Leningrad, Moscow, Voronezh, Yakutia), Slovakia, Spain, Sweden, Switzerland, Turkey (Antalya, Aydin, Denizli, Isparta, Kars, Mugla, Rize), UK, Ukraine; Afrotropical: South Africa, St. Helena. Multiregional polyzonal.

REMARKS. A record of the species from Yakutia needs confirmation as it can belong to H. callostomus Loew.

\section{Hydrophorus bipunctatus (Lehmann, 1822)}

REFERENCES. Grichanov, Bagachanova, 2006: 136 (C Yakutia: Lena-Amga interfluve, Lena River valley).

DISTRIBUTION. Type locality: Germany: Hamburg. Palaearctic: Austria, Belgium, Belarus, Bosnia and Herzegovina, Czech, Denmark, Estonia, Finland, France, Germany, Hungary, Ireland, Italy, Kyrgyzstan, Latvia, Netherlands, Norway, Poland, Romania, Russia (Buryatia, KarachaiCherkessia, Karelia, Kursk, Leningrad, Mordovia, Moscow, Ryazan, S Ural, Voronezh, Yakutia), Sweden, Switzerland, UK. Euro-Siberian-Central-Asian, temperate.

99. Hydrophorus brunneifacies Negrobov, 1977*

MATERIAL EXAMINED. NE Yakutia: $20^{7} q$, Cherskii, 18.08.1972, Gorodkov (ZIN).

DISTRIBUTION. Type locality: Kazakhstan: Shortandy, 70 km NW Tselinograd [=Astana]. Palaearctic: Kazakhstan, Russia (Altai Terr. [Makhlakovskoe], Yakutia). New for Yakutia.

HABITATS. Swamp, on water.

\section{Hydrophorus brunnicosus Loew, 1857}

REFERENCES. Negrobov, 1977: 368 (Yakutia); Negrobov, Chalaya, 1991: 76 (Yakutia: 12 versts downstream Zhigalov, 23.IX.1925). 
REMARKS. Old records of the species from Yakutia were based on material collected in 1925 in the Zhigalovskii district, now belonging to the Irkutsk Region.

100. Hydrophorus callostomus Loew, 1857

REFERENCES. Grichanov, 2002: 124 (C Yakutia: Lena-Amga interfluve); Grichanov, Bagachanova, 2006: 136 (C Yakutia: LenaAmga interfluve, Lena River valley).

MATERIAL EXAMINED. C Yakutia: $30^{7}+$, Amginsky District, Mikhailovka, 8.08.1987, Zlobin (ZIN); Lena River valley. $30^{7}$, Botanical Garden, 18.08.2005, Evdokarova (IBPC); 1 ㅇ, $35 \mathrm{~km} \mathrm{~N}$ vil Kapitonovka, 12.07.2007, Ermakova (IBPC); $10^{7}, 38 \mathrm{~km}$ SW Yakutsk, 16.08.2007, Ermakova (IBPC); 107, Elanka, 130 km SW Yakutsk, alas Ebe, 25.08.2005, 28.07.1977, Bagachanova (IBPC); $20^{\top}, 3$, Khatassy, pig farm, 16.07.2006, Bagachanova (IBPC); SW Yakutia: $30^{7}$ 29, Lena River left bank, 2nd Neryuktyaiinsk, $35 \mathrm{~km}$ SW Olekminsk, 23.07.2008, Bagachanova (IBPC); $10^{7}$, Biryuk River, 4 $\mathrm{km}$ from Melichan River mouth, 14.07.2008, Bagachanova (IBPC).

DISTRIBUTION. Type locality: Russia: Siberia. Palaearctic: Armenia, Kazakhstan, Russia (Adygea, Buryatia, Dagestan, Krasnodar, Leningrad, Omsk, Ural, Vladivostok, Voronezh, Yakutia, Yaroslavl), Sweden, Tajikistan, Turkey (Erzurum, Kars), Ukraine. Trans-Eurasian temperate. New for SW Yakutia.

HABITATS. Riparian communities, forb-cereal communities, branch at steppe slope, at lake, Carex, Sparganium, Stipa.

\section{Hydrophorus cinipunctus Negrobov, 1975*}

MATERIAL EXAMINED. W Yakutia: $10^{7}$, Kempendyai River, $75 \mathrm{~km}$ E vil. Suntar, 13.08.1988, Blagoderov (ZIN).

DISTRIBUTION. Type locality: Russia: "Umgebung von Petropawlowsk-Kamtschatskij Nogomy”. Palaearctic: Mongolia, Russia (Buryatia, Kamchatka, Yakutia). New for Yakutia.

102. Hydrophorus freyi Storå, 1954*

MATERIAL EXAMINED. C Yakutia: Lena-Vilyui interfluve, $10^{7}$, Labyda Lake, $17 \mathrm{~km}$ NW vil. Sitte, 21.07.2012, Sivtseva (IBPC)

DISTRIBUTION. Type locality: Finland: Jacobstad. Palaearctic: Finland, Russia (Buryatia, Khabarovsk, Vladivostok, Yakutia), Sweden. Trans-Eurasian temperate. New for Yakutia.

HABITATS. Hummocky tundra, sedge-cereal meadow.

\section{Hydrophorus litoreus Fallén, 1823}

REFERENCES. Negrobov, 1975: 64 (no material provided).

MATERIAL EXAMINED. C Yakutia: Lena River valley, 29 , Oktemtsy, 5.06.1977, Maksimova (IBPC).

DISTRIBUTION. Type locality: Sweden: Balthici Esperod. Palaearctic: Austria, Belarus, Belgium, Czech, Denmark, Estonia, Finland, France, Germany, Hungary, Ireland, Latvia, Netherlands, Poland, Romania, Russia (Kamchatka, Karelia, Leningrad, Lipetsk, Moscow, Murmansk, Pskov, Tyumen, Voronezh, West Siberia, Yakutia), Slovakia, Sweden, UK, Ukraine (Uzhgorod). Trans-Eurasian temperate. First reliable record from Yakutia.

Hydrophorus nebulosus Fallén, 1823

REFERENCES. Grichanov, 2006: 32 (Yakutia).

REMARKS. Yakutia was in error included in the area of the species by Grichanov [2006] in his species list.

\section{Hydrophorus signifer Coquillett, 1899}

REFERENCES. Grichanov, Bagachanova, 2006: 135 (N Yakutia). MATERIAL EXAMINED. N Yakutia: $20^{7}+$, Saskylakh airport, 24.07.1988, Gorodkov (ZIN); NE Yakutia: $300^{7}+$, Cherskii, 18.08.1972, Gorodkov (ZIN).

DISTRIBUTION. Type locality: Russia: Bering Is. Palaearctic: Austria, Belgium, Czech, Finland, Latvia, Netherlands, Norway, Sweden, Russia (Chukotka, Karelia, Mur- mansk, Nenetsia, N Ural, Yakutia); Nearctic: USA, Canada. Holarctic Arctic-boreal. New for NE Yakutia.

HABITATS. Forest-tundra, swamp hollows, bank of lake; on water.

105. Medetera bargusinica Negrobov, 1972

REFERENCES. Grichanov, 2002: 124 (C Yakutia: Lena-Amga interfluve); Grichanov, Bagachanova, 2006: 137 (C Yakutia: LenaAmga interfluve).

MATERIAL EXAMINED. C Yakutia: Lena River valley: 40', 1 , , Tabaginskii cape, $38 \mathrm{~km}$ Pokrovsky road, Malaise trap, 1113.08.2010, Bagachanova (IBPC); Lena-Amga interfluve: $10^{7}$, Churapchinsky ulus, Mendige, 122 km E Yakutsk, 14.07.2004, Bagachanova (ZIN).

DISTRIBUTION. Type locality: Russia: "Burjatien, Transbaikalien, Jarechta". Palaearctic: Mongolia, Russia (Buryatia, Yakutia). Daurian-Mongolian, steppe inhabitant.

HABITATS. Steppe at slope, cereal-forb meadow, steppe meadow; Agropyron, Pedicularis.

106. Medetera bilineata Frey, 1915

REFERENCES. Negrobov, Chalaya, 1991: 77 (C Yakutia: Yakutsk).

DISTRIBUTION. Type locality: Finland: Karislojo, Sammatti, Pyhajarvi, Messuby, Systems, Muonio, Enonteris, Palmen, Kantalaks. Palaearctic: Czech, Finland, Kazakhstan, Russia (Arkhangelsk, Leningrad, Kursk, Voronezh, Perm, Yakutia). Euro-Siberian temperate.

REMARKS. Bickel [1985] considered this name as synonym of Nearctic and Neotropical Medetera veles Loew, 1861 , examining the holotypes of the two species. Nevertheless, Negrobov and Naglis [2016] raised M. bilineata from synonymy, mentioning the fine difference in setation of male epandrial lobe.

\section{Medetera jakuta Negrobov, 1972}

REFERENCES. Negrobov, Stackelberg, 1974: 310 (C Yakutia: Yakutsk); Negrobov, Chalaya, 1991: 77 (C Yakutia: Yakutsk).

DISTRIBUTION. Type locality: Russia: Yakutsk. Palaearctic: Russia (Yakutia). Conditional endemic, boreal.

\section{Medetera lamprostomoides Negrobov, 1972}

REFERENCES. Negrobov, Stackelberg, 1974: 313 (C Yakutia: Lena River valley; Lena-Amga interfluve); Negrobov, Chalaya, 1991: 77 (C Yakutia: Lena River valley).

MATERIAL EXAMINED. C Yakutia: 507, 5ㅇ, Chochur Muran, 10.07.2007, Bagachanova (IBPC).

DISTRIBUTION. Type locality: Russia: "Dorf Namskoe linkes Ufer des Fluses Lena, 90 km von Jakutsk". Palaearctic: Kazakhstan, Russia (Yakutia), Turkey (A ri, Kars). CentralAsian-Yakutian, steppe inhabitant.

HABITATS. Steppe slope, cereal-forb community.

\section{Medetera micacea Loew, 1857}

REFERENCES. Negrobov, Stackelberg, 1974: 318 (Yakutia). Negrobov, Chalaya, 1991: 77 (C Yakutia: Lena River valley); Grichanov, Bagachanova, 2006: 137 (C Yakutia: Lena-Amga interfluve, Lena River valley).

MATERIAL EXAMINED. C Yakutia: Lena River valley: 10", 1ㅇ, Yakutsk, 19-20.07.1987, Zlobin (ZIN); 107, Vladimirovka, 20 km SW Yakutsk, 28.06.07; $90^{7} 9,23 \mathrm{~km}$ SW Yakutsk, env. vil. Vladimirovka, 28.06.2007, Bagachanova (IBPC); $50^{7}+, 23 \mathrm{~km} \mathrm{SW}$ Yakutsk, 28.07.2007, 6.08.2007; 107, Breeding farm, $7 \mathrm{~km} \mathrm{SW}$ Yakutsk, 18.06.2008, Bagachanova (IBPC); $20^{\circ}$, Botanical Garden, Chochur Muran, 10.07.2007, Bagachanova (IBPC); $2 \sigma^{7}$, Botanical Garden, Chochur Muran, 3.07.2008, Ermakova (IBPC); 40 9 , 25 km N Yakutsk, env. vil. Kildyamtsy, 04.07.2007, Bagachanova (IBPC); $20^{7}+, 65 \mathrm{~km} \mathrm{SW}$ Yakutsk, 25.05.2007; Tabaginskii cape, 36 km SW Yakutsk, Malaise trap, 28-30.07.2009, Bagachanova 
(IBPC); $50^{\text {To }}+18$ km SW Yakutsk, 28.06.2007, Bagachanova (IBPC); $4 \sigma^{\gamma}, 1$, ,Tabaginskii cape, Malaise trap, 11-16.07.2010, Bagachanova (IBPC); 10 , 19, same label, 11-13.03.2010; 1ㅇ, Namski ulus, 1st Khomustakh, 29.06.2012; Nemyuginskii station YaNIISKh, 73 km SW Yakutsk, 30.06.20010, Evdokarova (IBPC); Amga River valley: $10^{7}$, Mikhailovka, $60 \mathrm{~km} \mathrm{~N} \mathrm{Amgi,} \mathrm{13.07.1985,} \mathrm{Mak-}$ simova (IBPC); 1을 Kharama Khaiata on Amga River near vil. Abaga, 20.07.2009, Bagachanova (IBPC); 207, 29, Amga River, env. vil. Syrdyk, $25 \mathrm{~km}$ from vil. Bolugur, 11-13.07.2009, Bagachanova (IBPC); SW Yakutia. 10 , 19, Biryuk River, $5 \mathrm{~km}$ from mouth, left bank, 18.07.2008; $1 \sigma^{\top}$, Biryuk River, $14 \mathrm{~km}$ from mouth, Lena River left tributary, 12.07.2008, Bagachanova (IBPC).

DISTRIBUTION. Type locality: not given [Europe]. Palaearctic: Austria, Belgium, Bosnia and Herzegovina, Bulgaria, China, Croatia, Denmark, Estonia, France, Germany, Greece, Hungary, Iran, Italy, Kazakhstan, Malta, Mongolia, Netherlands, Norway, Poland, Romania, Russia (Adygea, Chechnya, Crimea, Krasnodar, Leningrad, Omsk, Voronezh, Yakutia), Slovakia, Spain, Sweden, UK, Ukraine (Cherkasy), Uzbekistan. Euro-Siberian-Central-Asian temperate. New for SW Yakutia.

HABITATS. Steppe slope, dish, xerophyte meadow, cereal-forb community; Stipa, Koeleria, Melilotus, Carex, Potentilla, Thymus, Pulsatilla, Delphinium, Agrostis.

\section{Medetera truncorum Meigen, 1824}

REFERENCES. Grichanov, Bagachanova, 2006: 137 (C Yakutia: Lena-Amga interfluve).

DISTRIBUTION. Type locality: Germany: Hamburg. Palaearctic: Algeria, Austria, Azerbaijan, Belgium, Bosnia and Herzegovina, Czech, Croatia, Denmark, Egypt, Estonia, Finland, France, Germany, Greece, Hungary, Ireland, Israel, Luxembourg, Netherlands, Norway, Poland, Portugal incl. Azores, Russia (Krasnodar, Yakitia), Slovakia, Spain, Sweden, Switzerland, Turkey (Ad yaman, Gaziantep, Hatay, Isparta, Mersin, $\mathrm{Mu}$ la, anl urfa), UK, Ukraine (Kharkiv); Nearctic: British Columbia, Wyoming, Oregon. Holarctic polyzonal.

111. Medetera vagans Becker, 1917

REFERENCES. Negrobov, 1971: 45 (C Yakutia: Lena-Amga interfluve); Negrobov, Chalaya, 1991: 77 (NE Yakutia).

DISTRIBUTION. Type locality: Sweden: Lappland; Germany: Berlin. Palaearctic: Czech, Finland, France, Germany, Norway, Russia (Buryatia, Irkutsk, Karelia, Leningrad, Murmansk, Vladivostok, Yakutia), Slovakia, Sweden, Switzerland. Trans-Eurasian temperate.

\section{Medetera veles Loew, $1861^{*}$}

MATERIAL EXAMINED. SW Yakutia. 10', Lensky District, vil. Tolon, 13.07.1987, Zlobin (ZIN).

DISTRIBUTION. Type locality: USA: Florida. Palaearctic: Russia (Karelia, Khantia-Mansia, Yakutia), Spain; Nearctic: throughout Canada and USA, Bermuda, Mexico). Multiregional polyzonal.

REMARKS. Previous records of the species from Yakutia belong to Medetera bilineata Frey (see above), following synonymy proposed by Bickel (1985).

\section{Melanostolus sp.}

MATERIAL EXAMINED. W Yakutia: 10", env. Tumul, 60 $\mathrm{km} \mathrm{N}$ vil. Suntar, Kyukei Lake, N 62॰43'38", E 11745'44", 27.06.2011, L. Sivtseva (ZIN).

REMARKS. The male examined belongs most probably to an undescribed species.

\section{Melanostolus nigricilius (Loew, 1871)}

REFERENCES. Grichanov, 2002: 124 (C Yakutia: Lena-Amga interfluve), Grichanov, Bagachanova, 2006: 137 (C Yakutia: LenaAmga interfluve).
MATERIAL EXAMINED. C Yakutia: $1 \sigma^{7}$, Lena-Amga interfluve, alas Alagar-Khoi, $18 \mathrm{~km} \mathrm{~N}$ vil. Tyungyulyu, 21.07.1991, Bagachanova (ZIN).

DISTRIBUTION. Type locality: Tajikistan. Palaearctic: Bulgaria, China, France, Germany, Hungary, Israel, Mongolia, Romania, Russia (Yakutia), Tajikistan. Euro-SiberianCentral-Asian temperate.

\section{Micromorphus jacutensis Negrobov, 2000}

REFERENCES. Negrobov, 2000: 23 (?W Yakutia).

DISTRIBUTION. Type locality: Russia: Yakutia. Palaearctic: Russia (Yakutia). Conditional endemic, boreal.

116. Peodes yeniseiensis Grichanov, 2012*

MATERIAL EXAMINED. NE Yakutia: 10', 1, Cherskii, River bank, 6.08.1972, Gorodkov (ZIN).

DISTRIBUTION. Type locality: Russia: Krasnoyarsk Territory, Taymyrskiy Dolgano-Nenetskiy District, Dudinka env., Nikandrovskiy Island. Palaearctic: Russia (Taymyria, Yakutia). East-Siberian, Arctic-boreal. New for Yakutia.

\section{Rhaphium basale Loew, 1850}

REFERENCES. Negrobov, 1976a: 46 (C Yakutia; W Yakutia). MATERIAL EXAMINED. C Yakutia: 107, 19, Yakutsk, 21.07.1987, Zlobin (ZIN).

DISTRIBUTION. Type locality: Poland: "Schlesien". Palaearctic: Austria, Finland, Poland, Russia ("Central European Russia", Saratov, N Ural, Krasnoyarsk, Yakutia), Sweden. Euro-Siberian, boreal-montane.

118. Rhaphium commune (Meigen, 1824)

REFERENCES. Negrobov, 1979: 490 (C Yakutia).

DISTRIBUTION. Type locality: ?Germany: ?Aachen. Palaearctic: Austria, Belgium, Bosnia and Herzegovina, Czech, Denmark, Estonia, Finland,France, Germany, Hungary, Ireland, Italy, Latvia, Luxembourg, Netherlands, Norway, Poland, Russia (Adygea, Arkhangelsk, Crimea, Kamchatka, Karelia, Khabarovsk, Krasnodar, Krasnoyarsk, Leningrad, Moscow, Murmansk, Pskov, Voronezh, Yakutia), Romania, Slovakia, Spain, Sweden, Switzerland, UK; Nearctic: North America. Holarctic, ?boreal.

\section{Rhaphium crassipes (Meigen, 1824)*}

REFERENCES. Negrobov, 1979: 490 (Yakutia); Negrobov, Chalaya, 1991: 77 (C Yakutia: Lena River valley).

MATERIAL EXAMINED. C Yakutia: $20^{7}$, Middle Lena River, Tabaginskii cape, Malaise trap, 23-25.06.2010, Bagachanova (IBPC); 107, Yakutsk, 21.07.1987, Zlobin (ZIN); W Yakutia: $20^{7}$, Leninsky [=Nyurbinsky] District, Nyurba, 18, 30.06.1987, Zlobin (ZIN).

DISTRIBUTION. Type locality: not given. Palaearctic: Austria, Belgium, Czech, Denmark, Estonia, Finland, France, Germany, Hungary, Ireland, Italy, Latvia, Netherlands, Norway, Poland, Romania, Russia (Adygea, Alania, Arkhangelsk, Baikal, Irkutsk, Kamchatka, Karachai-Cherkessia, Karelia, Krasnodar, Leningrad, Lipetsk, Mordovia, Moscow, Murmansk, Nenetsia, Voronezh, Vladivostok, Yakutia, Yamalia), Slovakia, Sweden, Switzerland, UK; Nearctic: Canada: Yukon Terr., Northwest Terr., British Columbia, Alberta to Quebec; USA: Alaska. Holarctic temperate. New for W Yakutia.

HABITATS. Steppe slope, Agropyron.

\section{Rhaphium dichromum Negrobov, 1976*}

MATERIAL EXAMINED. N Yakutia: 10', Kyusyur, Lena River lower reaches, $12 \mathrm{~km}$ NE, Kyuryu-Myuyus river bank, $63 \mathrm{~m}$, 21.07.1957, Gorodkov (ZIN).

DISTRIBUTION. Type locality: Russia: Buryat ASSR, Barguzin reservation. Palaearctic: Russia (Altai Rep., Buryatia, Magadan). Siberian Arctic-Boreal. New for Yakutia. 
121. Rhaphium discolor Zetterstedt, 1838*

Porphyrops riparia Parent, 1925 (nec Meigen, 1824; misidentification).

REFERENCES. Stackelberg, 1962: 302 (Yakutia; as Porphyrops riparia $\mathrm{Mg}$.).

MATERIAL EXAMINED. C Yakutia: 10 , Oktemtsy, 5.06.1977, Bagachanova (IBPC).

DISTRIBUTION. Type locality: Sweden: "Lapponia Umensi; Lycksele; ripa lacus Borgsjoe; Asele; Lapponia meridionalis". Palaearctic: Austria, Belarus, Belgium, Denmark, Estonia, Finland, France, Germany, Ireland, Kirgizia, Latvia, Mongolia, Netherlands, Norway, Poland, Russia (Karelia, Leningrad, Murmansk,), Sweden, UK; Nearctic: USA: Alaska. Multiregional polyzonal. First reliable record from Yakutia.

122. Rhaphium elegantulum (Meigen, 1824)

REFERENCES. Negrobov, Chalaya, 1991: 78 (C Yakutia: Lena-Amga interfluve).

MATERIAL EXAMINED. C Yakutia: Lena River valley: $10^{7}$ Tabaginskii cape, 38 ề Pokrovsky road, Malaise trap, 23-25.06.2010; $10^{7}$, same label, 11-13.08.2010, Bagachanova (IBPC).

DISTRIBUTION. Type locality: Germany: Hamburg. Palaearctic: Austria, Belarus, Belgium, Czech Republic, Denmark, Estonia, Finland, France, Germany, Ireland, Latvia, Moldova, Netherlands, Norway, Poland, Romania, Russia (Kamchatka, Karelia, Khantia-Mansia, Krasnoyarsk, Krasnodar, Leningrad, Murmansk, Novosibirsk, Voronezh, Baikal, "Polar Ural", "Igarka"), Spain, Sweden, UK; Nearctic: USA and Canada: Alaska, Oregon, Idaho, Colorado, Quebec to Nova Scotia and New Brunswick, southward to New York and New Hampshire. Multiregional polyzonal.

HABITATS. Slope; forb meadow at water.

123. Rhaphium fasciatum Meigen, 1824*

MATERIAL EXAMINED. SW Yakutia: 10", Lensky District, vil. Tolon, 16.07.1987, Zlobin (ZIN).

DISTRIBUTION. Type locality: not given. Palaearctic: Austria, Belgium, Czech, Denmark, Estonia, Finland, France, Germany, Ireland, Latvia, Netherlands, Norway, Poland, Romania, Russia (Kabardino-Balkaria, Karachai-Cherkessia, Leningrad, Pskov, "Siberia"), Slovakia, Sweden, Switzerland, UK. Euro-Siberian temperate. New for Yakutia.

124. Rhaphium glaciale (Ringdahl, 1920)

REFERENCES. Negrobov, 1976a: 46 (C Yakutia: Lena-Amga interfluve); Negrobov, 1979: 502 (Yakutia).

MATERIAL EXAMINED. NE Yakutia: $30^{7}$, Cherskii, River bank, 6.08.1972, Gorodkov (ZIN).

DISTRIBUTION. Type locality: Sweden: Lapland, nr Torneträsk; Jämtland, Wällista. Palaearctic: Finland, Norway, Russia (Murmansk, Baikal, Khantia-Mansia, Krasnoyarsk, Magadan, Yakutia), Sweden; Nearctic: "North America". Holarctic, boreal. New for NE Yakutia.

125. Rhaphium latimanum Kahanpää, 2007

REFERENCES. Negrobov, Grichanov, 2010: 121 (C Yakutia Lena River valley).

DISTRIBUTION. Type locality: Finland: Kilpisjarvi. Palaearctic: Finland, Russia (Khantia-Mansia, Magadan, Yamalia, Yakutia). Euro-Siberian boreal.

\section{Rhaphium nasutum Fallén, 1923*}

REFERENCES. Grichanov, 2006: 47 (Yakutia; no materia provided).

MATERIAL EXAMINED. C Yakutia: $20^{7}$, Oktemtsy, island of Lena River, 8.07.1977, Maksimova (IBPC); SW Yakutia: 107, env. vil. Solyanka, $5 \mathrm{~km}$ N Olekminsk, 25.07.2008, Bagachanova
(IBPC); $10^{\prime}, 1$, Tabaginskii cape, $38 \mathrm{~km} \mathrm{SW}$ Yakutsk, Malaise trap, 28-30.07.2009, Bagachanova (ZIN).

DISTRIBUTION. Type locality: Sweden: "Svecia meridionali". Nearctic: Canada, USA: AK, WA, AB, ON, QC; Palaearctic: Austria, Belarus, Denmark, Finland, Belgium, Czech, France, Germany, Hungary, Kazakhstan, Latvia, Netherlands, Norway, Poland, Romania, Russia (Irkutsk, Karelia, Krasnoyarsk, Leningrad, Moscow, Ryazan, Tyumen, Voronezh), Slovakia, Sweden, UK. Multiregional. First reliable record from Yakutia.

HABITATS. Slope at branch; meadow; sedge.

\section{Rhaphium nudiusculum Negrobov, 1976}

REFERENCES. Negrobov, Chalaya, 1991: 78 (NE Yakutia).

DISTRIBUTION. Type locality: Russia: Buryat ASSR, Barguzin reservation. Palaearctic: Russia (Buryatia, Yakutia). East-Siberian boreal.

128. Rhaphium patellitarse (Becker, 1900)

REFERENCES. Negrobov, 1979: 502 (C Yakutia); Negrobov, Chalaya, 1991: 78 (C Yakutia: Lena River valley).

DISTRIBUTION. Type locality: Russia: Khantaika [Taimyr]. Palaearctic: Russia (Altai Rep., Buryatia, Taimyr, Yakutia, Yamalia). Siberian boreal.

Rhaphium riparium (Meigen, 1824)

REFERENCES. Stackelberg, 1962: 302 (Yakutia; as Porphyrops riparia $\mathrm{Mg}$.).

REMARKS. The record belongs to Rhaphium discolor (Meigen, 1824) (see above).

\section{Rhaphium rivale (Loew, 1869)}

REFERENCES. Stackelberg, 1962: 302 (Yakutsk; as Porphyrops rivalis Lw.); Negrobov, 1979: 502 (C Yakutia).

MATERIAL EXAMINED. W Yakutia: 10 $\sigma^{7}$, Leninsky [=Nyurbinsky] District, Nyurba, 30.06.1987, Zlobin (ZIN).

DISTRIBUTION. Type locality: Germany: "bei Langenau in der Grafschaft Glatz". Palaearctic: Austria, Belgium, Czech, Estonia, Finland, France, Germany, Latvia, Norway, Poland, Romania, Russia (Karelia, Krasnoyarsk, Leningrad, Pskov, Yakutia), Slovakia, Sweden, Switzerland, UK. New for W Yakutia. Euro-Siberian boreal-montane.

130. Rhaphium umbripenne (Frey, 1915)

REFERENCES. Negrobov, Chalaya, 1991: 78 (C Yakutia: Lena River valley).

MATERIAL EXAMINED. SW Yakutia: $10^{7}$, Lensky District, vil. Tolon, 16.07.1987, Zlobin (ZIN).

DISTRIBUTION. Type locality: Finland: Tavastehus, Kuopio, Tuovilanlahti, Ilomantsi, Jacobstad, Saraisniemi, Kuusamo, Muonio, Enontekis, Kantalaks, Ponoj, Fl. Voronje; Russia: Kola Peninsula. Nearctic: "North America"; Palaearctic: Finland, Norway, Russia (Kamchatka, Karelia, Khantia-Mansia, Leningrad, Murmansk, Yakutia), Sweden. Holarctic Arctic-boreal. New for SW Yakutia.

\section{Scellus alactaga Stackelberg, 1951}

REFERENCES. Stackelberg, 1951: 598 (C Yakutia: Lena River valley).

DISTRIBUTION. Type locality: Russia: Yakutia, 2 Neryukteiski nasleg, Padun by river Tunguska, lake Ivan $45 \mathrm{~km}$ NE Chita; Mongolia: Transbaikalia, river Tola, env. UlanBator. Palaearctic: Mongolia, Russia (Chita, Yakutia). Daurian-Mongolian, steppe inhabitant.

\section{Scellus gallicanus Becker, 1909}

REFERENCES. Stackelberg, 1951: 603 (C Yakutia: LenaAmga interfluve); Negrobov, Chalaya, 1991: 79 (C Yakutia: Lena- 
Amga interfluve; Lena River vallev); Grichanov, 2002: 124 (C Yakutia: Lena-Amga interfluve); Grichanov, Bagachanova, 2006 138 (NE Yakutia; C Yakutia: Lena-Amga interfluve).

MATERIAL EXAMINED. C Yakutia: $1 \sigma^{\prime}, 1+$, Yakutsk, Botanical Garden, 20.07.1987, Zlobin (ZIN)

DISTRIBUTION. Type locality: France: Lautaret. Palaearctic: China, France, Mongolia, Russia (Altai Rep., Murmansk, Sayan Mnts., Irkutsk, Yakutia). Euro-Siberian-Central-Asian temperate.

133. Scellus spinimanus (Zetterstedt, 1843)

REFERENCES. Stackelberg, 1951: 603 (C Yakutia); Negrobov, Chalaya, 1991: 79 (C Yakutia: Lena River valley; Amga River valley; Lena-Amga interfluve); Grichanov, Bagachanova, 2006: 138 (N Yakutia, NE Yakutia, C Yakutia: Lena River valley).

MATERIAL EXAMINED. NE Yakutia: Oimyakon env., summer station Taiga, $12 \mathrm{~km}$ from vil. Tomtor, 19.07.1979, Bagachanova (IBPC); 20', 29, Kolyma River, Pomazkino, 126 km NE Srednekolymsk, 1, 2, 3.08.2006, Popov (IBPC); 2 , Kolyma River, Lobuia station, 20 km NE Srednekolymsk, Bagachanova (IBPC); 207, Cherskii Ridge, Moma River head, $50 \mathrm{~km}$ E Pobeda Mnt., env. vil. Sasyr, 28.07.1998, Nogovitsyna (IBPC) $40^{7}, 39$, vil. Khaiysardakh, 38 km NE Batagai vil., 1.07.2009, Potapova (IBPC); $20^{7}, 1.5 \mathrm{~km}$ from rivulet Khara-Khaya, old Magadan road, N 6311'57", E 144º1'03", 18.07.2011, Bagachanova (IBPC); W Yakutia: $17 \sigma^{7}$, , vil. Arylakh SE Mirnyi, 8 , 9.07.1987, Zlobin (ZIN); $10^{\top}$, Nyurba, 6.08.1989, Bagachanova (IBPC); C Yakutia: Amga River valley: 1요 Amginsky District, Mikhailovka, 5.08.1987, Zlobin (ZIN); Lena River valley: $10^{7}$ Oktemtsy, 20.07.1978, Kaimuk (IBPC); SW Yakutia: $10^{7}$, Lensky District, vil. Tolon, 16.07.1987, Zlobin (ZIN). NE Yakutia. 1․ Cherskii, floodplain Kolyma River, 4.07.1971, Chelnokov (ZIN); N Yakutia: $160^{7}+$, Chekurovka, Lena River lower reaches, 24.07.1957, Gorodkov (ZIN); NW Yakutia: $1 \sigma^{7}$, Saskylakh, 25.07.1988, Gorodkov (ZIN)

DISTRIBUTION. Type locality: Scandinavia. Palaearctic: Austria, Finland, Mongolia, Norway, Sweden, Russia (Crimea, N Ural, Yakutia); Nearctic: Canada, USA: Alaska, Newfoundland, Manitoba, Northwest Terr., Yukon. Holarctic temperate. New for SW and W Yakutia.

HABITATS. Hummocky tundra, slope.

134. Sciapus vladimiri Grichanov et Negrobov, 2014

REFERENCES. Grichanov, Negrobov, 2014: 84 (C Yakutia: Yakutsk)

DISTRIBUTION. Type locality: Yakutia, Yakutsk, botanical garden. Palaearctic: Russia (Yakutia). Conditional endemic

135. Sympycnus changaicus Negrobov 1973

Sympycnus urgaicus Negrobov, 1973: 152.

REFERENCES. Grichanov, 2002: 124 (as Sympycnus urgaicus Negrobov, 1973: C Yakutia: Lena-Amga interfluve); Grichanov, Bagachanova, 2006: 139 (as Sympycnus urgaicus Negrobov, 1973, C Yakutia: Lena River valley, Lena-Amga interfluve); Negrobov et al. 2017: 532 (C Yakutia, NE Yakutia)

MATERIAL EXAMINED. NE Yakutia: 4ð', Yakutia, Batagai, swampy lake bank, 9.08.1972, Gorodkov (ZIN); 107, Yakutia, Verkhoyansk, bog, 25.07.1974, Gorodkov (ZIN); W Yakutia: 1\%', 19 (in copula), $1 \sigma^{\prime}$, env. Mirnyi, collector Yurelyakh, 14.07.2001, Nogovitsyna, Popov (IBPC); 707, 2 \%, vil. Tumul env., 60 km N vil. Suntar, Kyukei Lake, N 6243'38', E 11745'44", 17.06.2011, Sivtseva (IBPC); 1 웅 Mokhsogollokh salt lake, env. vil. Kempendyai, $61 \mathrm{~km}$ E vil. Suntar, N 6201'62", E 118³9'07", 23.06.2011, Sivtseva (IBPC); C Yakutia: Lena River valley: 10', Yakutsk, 18.VII.1969, Sychevskaya (ZIN); 1 , 18 km SW Yakutsk, 28.06.2007, Bagachanova (IBPC); 10'; Tabaginskii cape, 38 km SW Yakutsk, preventorium, 28.07.2009, Bagachanova (IBPC); 4 $\sigma^{\top}, 1$, , Khatassy, pig farm, khvoshch, 16.07.2008, Bagachanova (IBPC); 1ㅇ, Tabaginskii cape, 38 km Pokrovsky road, branch, Malaise trap, 11-13.08.2010, Bagachanova (IBPC); SW Yakutia: 1우, $2+$, Biryuk River mouth, 11.07.2008; 1 을 Neryuktyaiinsk, 35 km SW Olekminsk, 23.07.2008, Bagachanova (IBPC).
DISTRIBUTION. Type locality: Mongolia: “Archangaj aimak, Gebirgspas Egijn davaa". Palaearctic: Mongolia, Russia (Altai Rep., Buryatia, Chita, Yakutia). Daurian-Mongolian, steppe.

HABITATS. Steppe slope, forb meadow, cereal-forb hygrophyte meadow, swampy cereal meadow, at lake; shortawned barley, Carex, Ranunculus, Scolochloa, Sparganium.

\section{Sympycnus olejnicheki}

Negrobov, Barkalov et Selivanova 2013

Sympycnus spiculatus Gerstäcker: Grichanov, Bagachanova, 2006: 139 (misidentification).

REFERENCES. Grichanov, Bagachanova, 2006: 139 (as S. spiculatus Gerstäcker; N Yakutia); Negrobov et al., 2017: 538 (N Yakutia).

MATERIAL EXAMINED. NE Yakutia: 10', Momskij district, Indigirka River, lower reaches of Ystan-Yuryakh River, 2.07.1976, V. Kovalev leg. (ZIN); $1 \sigma^{7}$, Indigirka River, lower reaches of the In'jali River, 19.08.1976, V. Kovalev leg. (ZIN); $1 \sigma^{\text {T}, ~ V e r k h o j a n s k ~}$ settlement, Yana River bank, 13.08.1971, K. Gorodkov leg. (ZIN).

DISTRIBUTION. Type locality: Russia: Krasnoyarskij Kraj: East Taimyr, bank of river Zakharova Rassokha. Palaearctic: Russia (Taimyria, Yakutia). New for NE Yakutia. East-Siberian, Arctic-boreal.

HABITATS. Riverbank, on Salix sp.

\section{Sympycnus pulicarius (Fallén)}

REFERENCES. Negrobov et al., 2017: 538 (C Yakutia: Amga River valley; Lena River valley).

DISTRIBUTION. Type locality: Sweden. Palaearctic: Andorra, Austria, Azerbaijan, Belgium, Bosnia and Herzegovina, Bulgaria, Croatia, Czech, Denmark, Estonia, Finland, France, Georgia, Germany, Greece, Hungary, Iran, Ireland, Italy, Kazakhstan, Kyrgyzstan, Latvia, Lithuania, Luxembourg, Macedonia, Moldova, Mongolia, Netherlands, Norway, Poland, Portugal, Romania, Russia (Alania, Altai Rep., Altai Terr., Buryatia, Chechnya, Chelyabinsk, Crimea, Kabardino-Balkaria, Kaliningrad, Karachai-Cherkessia, Karelia, Khakassia, Krasnodar, Krasnoyarsk Terr., Leningrad, Lipetsk, Moscow, Murmansk, Novgorod, Pskov, Stavropol, Svedlovsk, Tambov, Voronezh, Yakutia), Serbia, Slovakia, Spain incl. Canary Islands, Sweden, Switzerland, Tajikistan, Turkey (Ayd n, Bolu, Mu la, Van), UK, Ukraine, Uzbekistan; Nearctic: California. Holarctic polyzonal.

\section{Sympycnus simplicitarsis Becker 1900}

REFERENCES. Negrobov et al., 2017: 538 (N, W and C Yakutia: Amga River valley, Lena-Amga interfluve, Lena River valley).

DISTRIBUTION. Type locality: Russia: Dudinka. Palaearctic: Russia (Altai Rep., Buryatia, Taimyria, Yakutia). East-Siberian boreal.

\section{Sympycnus taimyricus}

Negrobov, Barkalov, Selivanova et Grichanov 2016

Sympycnus brevimanus Loew: Grichanov, Bagachanova, 2006: 139 (misidentification)

REFERENCES. Grichanov, Bagachanova, 2006: 139 (as Sympycnus brevimanus Loew; N Yakutia).

DISTRIBUTION. Type locality: Russia: Taimyr Biosphere Reserve, Ary-Mas cordon. Palaearctic: Russia (Taymyria, Yakutia). East-Siberian, Arctic-boreal.

140. Sympycnus yakutensis

Negrobov, Grichanov et Selivanova, 2017

REFERENCES. Negrobov et al., 2017: 544 (C Yakutia: LenaAmga interfluve).

DISTRIBUTION. Type locality: Russia: Yakutia, LenaAmga interfluve, alas Alagar-Khoi, 18 km N Tyungyulyu vil. Palaearctic: Russia (Yakutia). Conditional endemic. 
141. Syntormon pumilus (Meigen, 1824)

REFERENCES. Grichanov, 2013: 112 (SW Yakutia).

MATERIAL EXAMINED. SW Yakutia: 207, Biryuk River, left tributary of Lena River, $14 \mathrm{~km}$ from mouth, 16.07.2008, Bagachanova (IBPC).

DISTRIBUTION. Type locality: not given. Palaearctic: Afghanistan, Armenia; Austria, Belarus, Belgium, Bulgaria, Czech, Denmark, ?Egypt; Estonia, Finland, France, Germany, Great Britain, Greece, Hungary, Ireland, ?Israel, Italy, Latvia, Morocco, Norway, Poland, Romania; Russia (Astrakhan, Kabardino-Balkaria, Kaluga, Karelia, Krasnodar, Kursk, Leningrad, Lipetsk, Moscow, Murmansk, Novosibirsk, Pskov, Ryazan, Stavropol, Vologda, Voronezh, Yakutia), Slovakia, Sweden, Spain (Canary Is.), Tunisia, Turkey (Erzurum, Kars, Korucuk), UK, Ukraine (Kherson, Odessa); Middle Asia. Trans-Palaearctic polyzonal.

HABITATS. Riparian community, Carex at spruce forest.

142. Tachytrechus notatus (Stannius, 1831)

REFERENCES. Grichanov, 2002: 124 (C Yakutia: Lena-Amga interfluve); Grichanov, Bagachanova, 2006: 139 (C Yakutia: LenaAmga interfluve).

DISTRIBUTION. Type locality: Germany: Hamburg. Palaearctic: Abkhazia, Armenia, Austria, Belgium, Bosnia and Herzegovina, Bulgaria, Czech, Denmark, Finland, France, Germany, Greece incl. Crete, Hungary, Iran, Ireland, Italy, Israel; Morocco, Netherlands, Norway, Poland, Romania; Russia (Adygea, Chechnya, Crimea, Kabardino-Balkaria, Krasnodar, Yakutia), Slovakia, Spain incl. Canary Is., Sweden, Switzerland, Syria, Turkey (Aydin, Korucuk, Mugla), Turkmenistan, UK. Western-Central-Palaearctic polyzonal.

\section{Thinophilus ruficornis (Haliday, 1838)}

REFERENCES. Grichanov, Bagachanova, 2006: 139 (C Yakutia: Lena-Amga interfluve).

DISTRIBUTION. Type locality: England: Tarbert. Palaearctic: Austria, Azerbaijan, Belgium, Bulgaria, China, Croatia, Czech, Denmark, Estonia, Finland, France, Germany, Hungary, Ireland, Italy, Kazakhstan, Kirgizia, Latvia, Mongolia, Netherlands, Norway, Poland, Portugal, Romania, Russia (Kabardino-Balkaria, Karelia, Murmansk, Rostov, Crimea, Novosibirsk, Omsk, Orenburg, Yakutia), Slovakia, Spain, Sweden, UK, Ukraine (Kherson, Odessa). EuroSiberian-Central-Asian temperate.

\section{Thrypticus atomus Frey, 1915}

REFERENCES. Negrobov, Stackelberg, 1971: 255 (Yakutia). DISTRIBUTION. Type locality: Finland: Karislojo. Palaearctic: Austria, Belgium, Czech, Finland, Hungary, Latvia, Netherlands, Sweden, Russia (Arkhangelsk, Leningrad, Pskov, Ryazan, Krasnoyarsk, Yakutia). Euro-Siberian borealmontane.

145. Thrypticus aff. cuneatus (Becker, 1917)

MATERIAL EXAMINED. C Yakutia: Lena-Amga interfluve: $30^{7}, 1$, , Tattinsky ulus, Khalaiamny, 183 km E Yakutsk, 11.07.2004, Bagachanova (ZIN).

HABITATS. Riparian community.

REMARKS. The material examined belongs most probably to an undescribed species.

146. Thrypticus emiliae Negrobov, 1971

REFERENCES. Grichanov, 2002: 125 (C Yakutia: Lena-Amga interfluve); Grichanov, Bagachanova, 2006: 139 (C Yakutia: LenaAmga interfluve).

MATERIAL EXAMINED. C Yakutia: Lena River valley: 10", 2 , , Enseli, 37 km NE vil. Namtsy, 23.07.2011, Ermakova (IBPC); 107,
2ㅇ, Khatassy, 7 km SW Yakutsk, pig farm, 16.07.2006, Bagachanova (IBPC); 10', Elanka, 28.06.2008, Bagachanova (IBPC); SW Yakutia: $10^{7}$, Lensky District, vil. Tolon, 14.07.1987, Zlobin (ZIN); 30 , 5웅, Kyachchi, 21 km S Olekminsk, 22.07.2008, Bagachanova (IBPC).

DISTRIBUTION. Type locality: Russia: "SO Altai, Kosch-Agatsch, das Tal des Flusses Tschuja". Palaearctic: Russia (Altai Rep., Yakutia). Altai-Yakutan, steppe. New for SW Yakutia.

HABITATS. Riparian community, cereal-forb community at spruce forest, swampy lake bank; meso-hygrophyte, hygrophyte communities.

147. Thrypticus intercedens Negrobov, 1967

REFERENCES. Grichanov, 2002: 125 (C Yakutia: Lena-Amga interfluve); Grichanov, Bagachanova, 2006: 139 (C Yakutia: LenaAmga interfluve).

MATERIAL EXAMINED. C Yakutia: Lena River valley: $30^{7}$, 2 , , Khatassy, 7 km SW Yakutsk, pig farm, 16.07.2006, Bagachanova (IBPC); SW Yakutia: 30', Biryuk River mouth, Lena River left tributary, 11.07.2008; 20, 1웅 Biryuk River, $4 \mathrm{~km}$ from Melichan River mouth, 14.07.2008; 10', Biryuk River, $14 \mathrm{~km}$ from mouth, branch, 16.07.2008, Bagachanova (IBPC).

DISTRIBUTION. Type locality: Russia: Borok, Jaroslavl' region. Palaearctic: Finland, France, Germany, Iran, Norway, Russia (Khantia-Mansia, Sayan Mnts., Yakutia, Yaroslavl), Sweden, Turkey (Hakkari). Euro-Siberian-WestAsian temperate. New for SW Yakutia.

HABITATS. Riparian community, sedge community at spruce forest, rever bank at slope; hygrophyte community, Carex, Eleohcara.

\section{Thrypticus aff. nigricauda Wood, 1913}

MATERIAL EXAMINED. C Yakutia: Lena-Amga interfluve: $20^{\top}$, Tattinsky ulus, Khalaiamny, 183 km E Yakutsk, 11.07.2004, Bagachanova (ZIN).

HABITATS. Riparian community.

REMARKS. The material examined belongs most probably to an undescribed species.

149. Thrypticus nigricauda Wood, $1913^{*}$

MATERIAL EXAMINED. W Yakutia: 207, Leninsky [=Nyurbinsky] District, Nyurba, 23.06.1987, Zlobin (ZIN); 10', 19, Suntarsky District, vil. Kutana, 2, 4.07.1987, Zlobin (ZIN).

DISTRIBUTION. Type locality: England: Norfolk. Palaearctic: Austria, Finnland, France, Germany, Hungary, Norway, Russia (Leningrad, Voronezh, Yakutia), Sweden. EuroSiberian temperate. New for Yakutia.

\section{Thrypticus pollinosus Verrall, 1912*}

MATERIAL EXAMINED. C Yakutia: Amga River valley: $10^{7}$, Amginsky District, Mikhailovka, 27.07.1987, Zlobin (ZIN).

DISTRIBUTION. Type locality: England: Aviemore, Devereux Pool in Herefordshire. Palaearctic: China, Finland, France, Germany, Netherlands, Sweden, Russia (Karelia, Leningrad, Ryazan, Voronezh, Yakutia), UK. New for Yakutia.

\section{Discussion}

As a result of our study, a new material of Dolichopodidae was collected and identified, and a revised check-list of Yakutian species was compiled. The present research gives new records, including 28 species found for the first time in Yakutia, 31 species recorded from new natural zones and four probably undescribed species. In total, 20 genera and 150 (including four unnamed) species are listed above that apparently makes 
up $60-70 \%$ of actual Dolichopodidae fauna in Yakutia. This paper provides also characteristics of Yakutian habitats for 54 species.

A preliminary analysis of the Dolichopodidae distribution in Yakutian natural zones has shown that the fauna of the Central Yakutia covered with plain middletaiga and fragments of relic steppe sites is most fully studied, with 110 species found and about 150 expected species number. Mountain territories of the Southern Yakutia, flat lands of Western and Southwestern parts of the Republic are undercollected, each with 10 to 30 known species and over 100 expected species number. The districts of Northern and Northwestern Yakutia numbers 13 and 10 species respectively. Mountains of the Northeast beyond the Verkhoyansk Range keep extensive sites of relic steppes and should have rich dolichopodid fauna, but only 30 species are revealed there so far.

The boreal element dominates among the Yakutian species of long-legged flies. As to the longitudinal ranges, among the 150 species found, 24 are Holarctic, 27 are Trans-Eurasian, 15 species are conditional endemics of boreal Yakutia. Among the other species, more than 20 ones have the easternmost point of distribution, nearly 20 ones are reported from the Far East and East Siberia, and about the same number of species are distributed in Yakutia and one or more adjacent regions of East and South Siberia. Nevertheless, many regions of the Far East and Siberia are insufficiently studied; therefore, any chorological scheme must be handled with care.

ACKNOWLEDGEMENTS. We are grateful to collectors of the material (IBPC) N.N. Vinokurov, N.K. Potapova, S.N Nogovitsyna, A.A. Popov, T.G. Evdokarova, Yu.V. Ermakova, L.G. Sivtseva, A.P. Burnashova, E.L. Kaimuk, and T.N. Maximova. The authors are sincerely grateful to Drs. E.P. Nartshuk, I.V. Shamshev and L.A. Kuznetsova (ZIN) for their kindness in providing specimens for this study. The work of AKB is partly supported by the IBPC Fundamental Basic Project No AAAA-A17-117020110058-4.

\section{References}

Bickel D.J. 1985. A revision of the Nearctic Medetera (Diptera: Dolichopodidae) // Technical Bulletin U. S. Department of Agriculture. No.1692. P.1-109.

Frey R. 1915. Diptera Brachycera aus den arktischen Kustgegenden Sibiriens // Scientific results of the Russian polar expedition of 1900-1903 under the command of Baron E.V. Toll. E: Zoology. Vol.2. No.10. P.1-35.

Gorodkov K.B. 1984. [Range types of insects of tundra and forests zones of European Part of USSR] // Arealy nasekomykh Evropeiskoi chasti SSSR. Leningrad. P.3-20 [in Russian].

Grichanov I.Ya. 2002. A check list of Yakutian Dolichopodidae (Diptera) // International Journal of Dipterological Research. Vol.13. No.2. P.121-125.

Grichanov I.Ya. 2006. A checklist and keys to North European genera and species of Dolichopodidae (Diptera). St.Petersburg: VIZR. P.1-120 (Plant Protection News Supplements).

Grichanov I.Ya. 2012. A new species of Dolichopus Latreille, 1796 from Turkey (Diptera: Dolichopodidae) // Caucasian Entomological Bulletin. Vol.8. No.2. P.316-318.
Grichanov I.Ya. 2013. Systematic notes on West-Palearctic species of the genus Syntormon Loew (Diptera: Dolichopodidae) // Grichanov I.Ya., Negrobov O.P. (Editors). Fauna and taxonomy ofDolichopodidae (Diptera). Collection of papers. St.Petersburg: VIZR RAAS (Plant Protection News Supplements ISSN 18153682). P.3-26.

Grichanov I.Ya. 2014. Alphabetic list of generic and specific names of predatory flies of the epifamily Dolichopodoidae (Diptera). St.Petersburg: VIZR. P.1-544 (Plant Protection News Supplements ISSN 1815-3682).

Grichanov I.Ya. 2017. Alphabetic list of generic and specific names of predatory flies of the epifamily Dolichopodoidae (Diptera). Second edition. St.Petersburg: VIZR. P.1-563. (Plant Protection News Supplements ISSN 1815-3682).

Grichanov I.Ya., Bagachanova A.K. 2006. New data on the distribution of Dolichopodidae (Diptera) in Yakutia (Siberia). // International Journal of Dipterological Research. Vol.17. No.2. P.127-140.

Grichanov I.Ya., Negrobov O.P. 1979. [Catalogue for the family Dolichopodidae (Diptera) in the USSR]. Voronezh: Voronezh Univ. P.1-128 [Dep. in VINITI, Moscow 04.02.80, No.417-80 dep.; in Russian].

Grichanov I.Ya., Negrobov O.P. 2014. Palaearctic species of the genus Sciapus Zeller (Diptera: Dolichopodidae). St.Petersburg: VIZR. P.1-84 p. (Plant Protection News Supplements ISSN 1815-3682).

Grichanov I.Ya., Volfov B.I. 2009. A new and rare species of the genus Campsicnemus Walker (Diptera, Dolichopodidae) in the fauna of Russia // Entomologicheskoe obozrenie. Vol.88. No.3. P.685-688 [in Russian; English translation: Entomological Review. Vol.89. No.8. P.962-964].

Kuznetsova L.V. 2005. [Floristic zoning] // Plant Diversity of Yakutia. Novosibirsk: Publ. SB RAS. P.13-41 [in Russian].

Maslova O.O. 2006. [Revision of the genus Chrysotus (Dolichopodidae, Diptera) of Russia and adjacent territories]. Abstract of PhD Thesis (MS). Voronezh: Voronezh LTA. P.1-20 [in Russian].

Maslova O.O., Negrobov O.P., Selivanova O.V. 2011. [Fauna of the genus Chrysotus Meigen (Diptera, Dolichopodidae) of Russia. Part 1. Species groups Ch. cilipes Meigen and Ch. laesus Wied.] // Entomologicheskoe obozrenie. Vol.90. No.2. P. 464-468 [in Russian].

Negrobov O.P. 1971. [Little-known species of subfamily Medeterinae (Diptera, Dolichopodidae) of USSR fauna] // Vestnik zoologii. No.5. P. 43-46 [in Russian].

Negrobov O.P. 1975. [Species of the genus Hydrophorus (Dolichopodidae, Diptera) of USSR fauna] // Problemy izucheniya i okhrany landshaftov. Voronezh. No.2. P.63-66 [in Russian].

Negrobov O.P. 1976a. [New and little-known species of family Dolichopodidae (Diptera) of fauna of the USSR and adjacent territories] // Nauchnye doklady vysshei shkoly, biologicheskie nauki. No.8. P. 45-50 [in Russian].

Negrobov O.P. 1976b. [Species of Dolichopus of subgenus Hydroceleuthus (Diptera, Dolichopodidae) of Eastern Palaearctic] // Nasekomye Mongolii. Leningrad. Vol.4. P.509-517 [in Russian].

Negrobov O.P. 1977. Dolichopodidae // E. Lindner. Die Fliegen der Paläarktischen Region. Bd. IV/5. Teil 29. Lief.316. P.347-386.

Negrobov O.P. 1979. Dolichopodidae // E. Lindner. Die Fliegen der Paläarktischen Region. Bd. IV/5. Teil 29. Lief.322. P. 475-530.

Negrobov O.P. 1986a. To the fauna of family Dolichopodidae (Diptera) of Amurskaya oblast of the USSR and Mongolia // Nauchnye doklady vysshei shkoly, biologicheskie nauki. No.10. P.35-38 [in Russian].

Negrobov O.P. 1986b. [To the fauna of family Dolichopodidae (Diptera) of Amurskaya oblast] // Trudy zoologicheskogo instituta AN SSSR. Vol.146. P.10-14 [in Russian].

Negrobov O.P. 2000. Revision of the Palearctic species of the genus Micromorphus Mik (Diptera, Dolichopodidae) // International Journal of Dipterological Research. Vol.11. No.1. P.19-26.

Negrobov O.P., Chalaya O.N. 1991. [Dolichopodidae (Diptera) of North-Eastern part of USSR] // Entomologicheskie issledovaniya na Severo-Vostoke SSSR. Vladivostok: DVO AN SSSR. P.66-80 [in Russian].

Negrobov O.P., Grichanov I.Ya. 2010. The Rhaphium crassipes species group in the Palearctic Region with the description of a 
new species from Uzbekistan (Diptera: Dolichopodidae) // Caucasian Entomological Bulletin. Vol.6. No.1. P.117-122.

Negrobov O.P., Grichanov I.Ya. 2013. On the distribution of Dolichopus humilis Van Duzee in the Palaearctic Region and a new synonym (Diptera: Dolichopodidae: Dolichopus latipennis species group) // Zootaxa. Vol.3599. No.5. P.499-500.

Negrobov O.P., Grichanov I.Ya., Barkalov A.V. 2009. The Dolichopus latipennis species group (=Hygroceleuthus Loew) in the Palaearctic Region (Diptera: Dolichopodidae) // Zootaxa. No. 2087. P.37-45.

Negrobov O.P., Grichanov I.Ya., Selivanova O.V. 2017. Review of East Palaearctic species of Sympycnus Loew, 1857, with a key to species // Zootaxa. Vol.4277. No.4. P.531-548.

Negrobov O.P., Maslova O.O. 1995. [Revision of Palaearctic species of genus Chrysotus Mg. (Diptera, Dolichopodidae). II] // Entomologicheskoe obozrenie. Vol.74. No.2. P.456-466 [in Russian].

Negrobov O.P., Mokeeva S.Yu., Maslova O.O., Selivanova O.V. 2000a. [To the study of the family Dolichopodidae (Diptera) in the Far East] // Bioraznoobrazie i dinamika ekosistem Severno Evrazii. Novosibirsk. Vol.3. Part 1. P.84-86 [in Russian].

Negrobov O.P., Naglis S. 2016. Palaearctic species of the genus Medetera (Diptera: Dolichopodidae) // Zoosystematica Rossica. Vol.25. No.2. P.333-379.

Negrobov O.P., Selivanova O.V., Maslova O.O., Chursina M.A 2013. Check-list of predatory flies of the family Dolichopodidae (Diptera) in the fauna of Russia // Grichanov I.Ya., Negrobov O.P. (eds.). Fauna and taxonomy of Dolichopodidae (Diptera). Collection of papers. St.Petersburg: VIZR RAAS (Plant Protection News Supplements ISSN 1815-3682). P.47-93.

Negrobov O.P., Stackelberg A.A. 1971. Dolichopodidae // E. Lindner. Die Fliegen der Paläarktischen Region. Bd. IV/5. Teil 29. Lief.284. P.238-256.

Negrobov O.P., Stackelberg A.A. 1974. Dolichopodidae // E. Lindner. Die Fliegen der Paläarktischen Region. Bd. IV/5. Teil 29. Lief.302. P.303-324.

Negrobov O.P., Tsurikov M.N. 1986. [Two new species of genus Chrysotus (Diptera, Dolichopodidae) from Yakutia] // Zoologicheskii zhurnal. Vol.65. No.4. P.632-634 [in Russian].
Negrobov O.P., Tsurikov M.N., Maslova O.O. 2000b. [Revision of Palaearctic species of genus Chrysotus Mg. (Diptera, Dolichopodidae). III]// Entomologicheskoe obozrenie. Vol.79. No.1. P.227-238 [in Russian].

Negrobov O.P., Zlobin V.V. 1978. [Review of species of genus Campsicnemus Walker (Dolichopodidae, Diptera) of USSR fauna] // Nauchnye doklady vysshei shkoly, biologicheskie nauki. No.1. P.51-59 [in Russian].

Selivanova O.V., Negrobov O.P. 2011. [A new species of the genus Dolichopus (Diptera, Dolichopodidae) from Yakutia] // Bulletin of Moscow Society of Naturalists, Biological Series. Vol.116. No.1. P.64-65 [in Russian].

Stackelberg A.A. 1928. Dolichopodiden-Studien. I. Die paläarktischen Dolichopus-Arten mit gelben Schenkeln und schwarzen Postocularcilien // Zoologischer Anzeiger. Bd.79. S.260-269.

Stackelberg A.A. 1929. Dolichopodiden-Studien. II. Neue oder wenig bekannte Dolichopus Arten aus Ost-Sibirien // Zoologischer Anzeiger. Vol.84. Nos7-8. S.169-180.

Stackelberg A.A. 1930a. Dolichopodidae// E. Lindner. Die Fliegen der Paläarktischen Region. Bd.IV/5. Teil 29. Lief.59. S.1-64.

Stackelberg A.A. 1930b. [Scientific results of Far-Eastern hydrofaunistic expedition of Zoological Museum in 1927] // Annuaire du Musée Zoologique de I'Académie des Sciences de l'URSS. Vol.31. P.135-163 [in Russian with Latin summary].

Stackelberg A.A. 1933. Dolichopodidae // E. Lindner. Die Fliegen der Paläarktischen Region. Bd. IV/5. Teil 29. Lief.71. S.65-128.

Stackelberg A.A. 1951. [Palaearctic species of genus Scellus Lw. (Diptera, Dolichopodidae)] // Entomologicheskoye obozreniye. Vol.31. Nos3-4. P.595-608 [in Russian].

Stackelberg A.A. 1962. [List of Diptera of the Leningrad Region. V. Dolichopodidae] // Trudy Zoologicheskogo Instituta AN SSSR. Vol.31. Moscow, Leningrad: AN SSSR Publ. P.280-317 [in Russian]

Suslov S.P. 1999. Physical geography of the USSR: Asian part [2nd edition]. Moscow: Gos. uchebno-pedag. izdatel. 712 p. [in Russian]

Vinokurov N.N., Kanyukova E.V., Golub V.B. 2010. Catalog of Heteroptera of Asian part of Russia. Novosibirsk: Nauka. 320 p. [in Russian]. 